\title{
Time-series and Phase-curve Photometry of the Episodically Active Asteroid (6478) Gault in a Quiescent State Using APO, GROWTH, P200, and ZTF
}

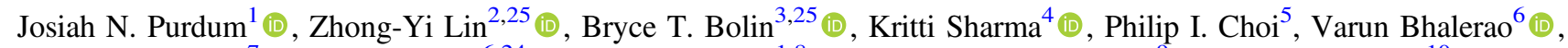
Josef Hanuš ${ }^{7}$ (i), Harsh Kumar ${ }^{6,24}$ (i), Robert Quimby ${ }^{1,8}$ (i), Joannes C. van Roestel ${ }^{9}$ (i) , Chengxing Zhai $^{10}$ (i),

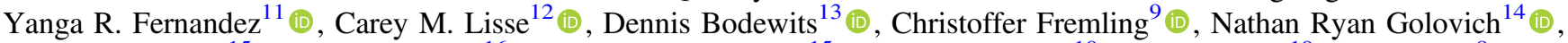
Chen-Yen $\mathrm{Hsu}^{15}$ (10), Wing-Huen $\mathrm{Ip}^{16}$, Chow-Choong Ngeow ${ }^{15}$ (1), Navtej S. Saini ${ }^{10}$, Michael Shao ${ }^{10}$, Yuhan Yao ${ }^{9}$ (1),

Tomás Ahumada $^{17}$ (1), Shreya Anand ${ }^{9}$ (1), Igor Andreoni ${ }^{9}$ (i), Kevin B. Burdge ${ }^{9}$ (1), Rick Burruss ${ }^{18}$, Chan-Kao Chang ${ }^{15}$ (1), Chris M. Copperwheat ${ }^{19}$ (i), Michael Coughlin ${ }^{20}$ (i), Kishalay De $^{9}$, Richard Dekany ${ }^{18}$ (1) Alexandre Delacroix ${ }^{18}$, Andrew Drake ${ }^{9}$, Dmitry Duev ${ }^{9}$ (10), Matthew Graham ${ }^{9}$ (10), David Hale ${ }^{18}$, Erik C. Kool ${ }^{21,22}$ (1), Mansi M. Kasliwal ${ }^{9}$ (1), Iva S. Kostadinova ${ }^{9}$,

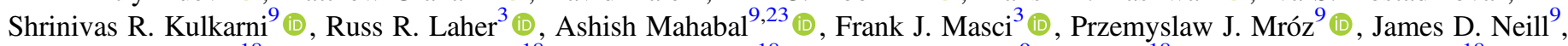
Reed Riddle ${ }^{18}$ (1) , Hector Rodriguez ${ }^{18}$, Roger M. Smith ${ }^{18}$ (i), Richard Walters ${ }^{9}$, Lin Yan ${ }^{18}$ (1) , and Jeffry Zolkower ${ }^{18}$

${ }^{1}$ Department of Astronomy, San Diego State University, 5500 Campanile Drive, San Diego, CA 92182, USA; jnpurdum@gmail.com ${ }^{2}$ Institute of Astronomy, National Central University, Taoyuan 32001, Taiwan

${ }^{3}$ IPAC, Mail Code 100-22, Caltech, 1200 E. California Boulevard, Pasadena, CA 91125, USA

${ }^{4}$ Department of Mechanical Engineering, Indian Institute of Technology Bombay, Powai, Mumbai-400076, India

5 Physics and Astronomy Department, Pomona College, 333 N. College Way, Claremont, CA 91711, USA

${ }^{6}$ Department of Physics, Indian Institute of Technology Bombay, Powai, Mumbai-400076, India

${ }^{7}$ Institute of Astronomy, Faculty of Mathematics and Physics, Charles University, V Holešovičkách 2, 18000 Prague, Czech Republic

${ }^{8}$ Kavli Institute for the Physics and Mathematics of the Universe (WPI), The University of Tokyo Institutes for Advanced Study, The University of Tokyo, Kashiwa, Chiba 277-8583, Japan

${ }_{10}^{9}$ Division of Physics, Mathematics and Astronomy, California Institute of Technology, Pasadena, CA 91125, USA

10 Jet Propulsion Laboratory, California Institute of Technology, 4800 Oak Grove Drive, Pasadena, CA 91109, USA

${ }^{11}$ Department of Physics and Florida Space Inst., University of Central Florida, 4000 Central Florida Boulevard, Orlando FL, 32816-2385, USA 12 Johns Hopkins University Applied Physics Laboratory, Laurel, MD 20723, USA

${ }_{13}$ Physics Department, Leach Science Center, Auburn University, Auburn, AL 36832, USA

${ }^{14}$ Lawrence Livermore National Laboratory, 7000 East Avenue, Livermore, CA 94550, USA ${ }^{15}$ Graduate Institute of Astronomy, National Central University, 32001, Taiwan

${ }^{16}$ Institute of Astronomy, National Central University, 32001, Taiwan

${ }^{17}$ Department of Astronomy, University of Maryland, College Park, MD 20742, USA

${ }^{18}$ Caltech Optical Observatories, California Institute of Technology, Pasadena, CA 91125, USA

${ }^{19}$ Astrophysics Research Institute, Liverpool John Moores University, 146 Brownlow Hill, Liverpool L3 5RF, UK

${ }^{20}$ School of Physics and Astronomy, University of Minnesota, Minneapolis, MN 55455, USA

${ }^{21}$ The Oskar Klein Centre, Department of Astronomy, Stockholm University, AlbaNova, SE-10691, Stockholm, Sweden

${ }^{22}$ Department of Physics and Astronomy, Macquarie University, NSW 2109, Sydney, Australia

${ }^{23}$ Center for Data Driven Discovery, California Institute of Technology, Pasadena, CA 91125, USA

Received 2021 February 20; revised 2021 March 17; accepted 2021 March 26; published 2021 April 26

\begin{abstract}
We observed the episodically active asteroid (6478) Gault in 2020 with multiple telescopes in Asia and North America and found that it is no longer active after its recent outbursts at the end of 2018 and the start of 2019. The inactivity during this apparition allowed us to measure the absolute magnitude of Gault of $H_{r}=14.63 \pm 0.02, G_{r}=0.21 \pm 0.02$ from our secular phase-curve observations. In addition, we were able to constrain Gault's rotation period using timeseries photometric lightcurves taken over $17 \mathrm{hr}$ on multiple days in 2020 August, September, and October. The photometric lightcurves have a repeating $\lesssim 0.05$ mag feature suggesting that (6478) Gault has a rotation period of $\sim 2.5 \mathrm{hr}$ and may have a semispherical or top-like shape, much like the near-Earth asteroids Ryugu and Bennu. The rotation period of $\sim 2.5 \mathrm{hr}$ is near the expected critical rotation period for an asteroid with the physical properties of (6478) Gault, suggesting that its activity observed over multiple epochs is due to surface mass shedding from its fast rotation spin-up by the Yarkovsky-O'Keefe-Radzievskii-Paddack effect.
\end{abstract}

Unified Astronomy Thesaurus concepts: Asteroids (72); Asteroid dynamics (2210); Main belt asteroids (2036)

Supporting material: machine-readable tables

\section{Introduction}

Active asteroids produce comet-like tails and comae that can be driven by many different types of forces different from the comets themselves (Jewitt et al. 2015). While sublimation of water ice is a primary driver for activity in "typical" comets, the $\sim 20$ known (so far) active asteroids in the main belt seem to

\footnotetext{
${ }^{24}$ LSSTC Data Science Fellow.

25 These authors contributed equally to this work.
}

lose mass via a wider array of physical effects such as collisions (e.g., Snodgrass et al. 2010), rotational instabilities (e.g., Jewitt et al. 2013), and thermal fracture (e.g., Jewitt et al. 2019a). We can assess the physics of a particular active asteroid's activity via observations over long time baselines that assess the object's photometric and morphological development. As more and more active asteroids are discovered, it is vital to continuously monitor these objects and determine the frequency of the various phenomena in the main belt. 
Table 1

Telescope Specifications and Parameters for This Work

\begin{tabular}{|c|c|c|c|c|c|c|}
\hline Telescope (1) & CCD (2) & Pixels (3) & Binning (4) & Scale $\left("\right.$ pix $\left.^{-1}\right)(5)$ & $\operatorname{Exp}(s)(6)$ & NST (7) \\
\hline MLO $1.0 \mathrm{~m}$ & ULTRAcam & $2 \mathrm{~K} \times 2 \mathrm{~K}$ & $2 \times 2$ & 0.358 & 120 & $\mathrm{~N}$ \\
\hline Lulin One-meter & SOPHIA & $2 \mathrm{~K} \times 2 \mathrm{~K}$ & $1 \times 1$ & 0.385 & 180 & $\mathrm{Y}$ \\
\hline ARC $3.5 \mathrm{~m}$ & ARCTIC & $2 \mathrm{~K} \times 2 \mathrm{~K}$ & $2 \times 2$ & 0.228 & 120 & $\mathrm{Y}$ \\
\hline Palomar 200 inch & CHIMERA & $1 \mathrm{~K} \times 1 \mathrm{~K}$ & $1 \times 1$ & 0.29 & 90 & $\mathrm{Y}$ \\
\hline Palomar 48 inch & ZTF CCD & $166 \mathrm{~K} \times 6 \mathrm{~K}$ & $1 \times 1$ & 1.01 & 30 & $\mathrm{~N}$ \\
\hline GIT $0.7 \mathrm{~m}$ & Apogee KAF3200EB & $2 \mathrm{~K} \times 1 \mathrm{~K}$ & $1 \times 1$ & 0.3 & $120-180$ & $\mathrm{~N}$ \\
\hline TMO $1.0 \mathrm{~m}$ & sCMOS & $1.6 \mathrm{~K} \times 1.6 \mathrm{~K}$ & $1 \times 1$ & 0.225 & 60 & $\mathrm{~N}$ \\
\hline
\end{tabular}

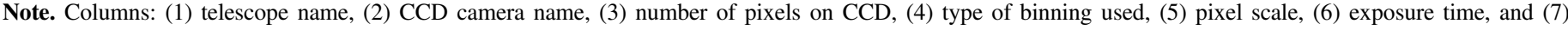
nonsidereal tracking enabled $(\mathrm{Y} / \mathrm{N})$.

The main belt asteroid (6478) Gault (1998 $\mathrm{JC}_{1}$; "Gault" hereafter) has been the subject of wide interest since the discovery in early 2019 of a comet-tail-like extended emission (Smith et al. 2019). Eventually, three tails were noted in 2019 January (Jewitt et al. 2019b; Ye et al. 2019) on the S-type Phocaea family member (Sanchez et al. 2019), suggesting multiple sporadic outbursts of activity. Ye et al. (2019) assessed the dynamics of the dust seen near Gault and estimated that two outbursts had actually occurred in late 2018. Searches through archival data that serendipitously caught Gault revealed that there had been active episodes in 2013, 2016, and 2017 as well (Chandler et al. 2019).

Many authors have proposed that the cause of Gault's activity is the instability of material on its surface (Hui et al. 2019; Jewitt et al. 2019b; Kleyna et al. 2019; Moreno et al. 2019; Ye et al. 2019) due to its rotation being spun up by the Yarkovsky-O'Keefe-Radzievskii-Paddack (YORP) effect (Bottke 2006; Kleyna et al. 2019). A critical observational test of this hypothesis would be to measure the asteroid's rotational period. Unfortunately, due to the dust around the asteroid, the rotational period had not been well constrained as reflected sunlight from dust grains would swamp the signal from the asteroid itself and thus suppress short-term lightcurve variations due to Gault's shape, as Jewitt et al. (2019b) indeed concluded. However, there have been published reports of some hints of rotational signatures in lightcurve data. For example, Ivanova et al. (2020) suggest a rotation period of 1.79 hr, Carbognani \& Buzzoni (2020) suggest 3.34 hr, Ferrín et al. (2019) have $3.36 \mathrm{hr}$, and Kleyna et al. (2019) suggest $\sim 2$ hr. In all cases, the lightcurve amplitude was quite small, on the order of just a few hundredths of a magnitude, which would be on the same order as the signal noise. It should also be noted that others (e.g., Moreno et al. 2019) report no variation in photometry over a time span longer than these periods. This very small amplitude demonstrates the challenge of photometrically extracting a rotation period from an active body (see also Bolin \& Lisse 2020, for an example).

In this paper, we report on several sets of imaging and photometry of Gault obtained in 2019 while it was still active as well as in 2020 when the asteroid appeared to be quiescent (Purdum et al. 2020). These data sets have allowed us to constrain the rotation period of Gault. We also use all of the data to understand the longer-term, secular variations in Gault's activity. In Section 2, we describe the observations from 2019 and 2020. In Section 3, we present the photometry, and in
Section 4, we discuss Gault's behavior, spin state, and shape, and summarize the article.

\section{Observations}

For our analysis, we made use of both our own, PI-led, pointed observations using several telescope facilities in the GROWTH (Global Relay of Observatories Watching Transients Happen) network (Kasliwal et al. 2019) and other faculties, as well as archival data from the Zwicky Transient Survey (ZTF; Bellm et al. 2019; Graham et al. 2019). Our pointed observations occurred on 17 nights between 2019 January 8 and 2020 October 20 and made use of six telescopes. On 7 of those 17 nights, we were able to have multiple telescopes follow Gault in a coordinated effort. The observations in 2019 showed Gault to still be active, but all such observations in 2020 showed no activity, only a point source (Purdum et al. 2020). The ZTF data are from 89 nights from 2018 November 1 to 2020 October 14. All data in this work have been flat- and bias-corrected because only CCDs were used. Tables 1 and 2 list the technical details of each telescope and the particulars about each observing run, respectively, while Tables 3 and 4 are the photometric data plotted for this work and the archival ZTF survey, respectively. We describe below each telescope facility used in our work.

\subsection{Mount Laguna Observatory $1.0 \mathrm{~m}$ Telescope}

Images of Gault were taken with the Mount Laguna Observatory (MLO) $1.0 \mathrm{~m}$ telescope on 2020 June 24 UT, several months after the asteroid was leaving solar conjunction. A total of 120 images were taken with $30 \mathrm{~s}$ exposure times each, culminating in 60 minutes of total exposure to measure the morphology of the asteroid.

Later observations were taken of Gault with MLO with an aim to constrain a rotation period. These data were taken between 2020 August 23 UT and 2020 October 20 UT over six separate campaigns listed in Table 2. MLO lightcurve images were taken in the Johnson-Cousins $R$ filter with between 165 and 190 separate $120 \mathrm{~s}$ exposures.

\subsection{Lulin One-meter Telescope}

The time-series observations of Gault using the Lulin onemeter Telescope (LOT) at the Lulin Observatory, Taiwan, for 2020 August 23 and 24 UT lasted $6.4 \mathrm{hr}$ and $5.2 \mathrm{hr}$, respectively. The other time span on 2020 September 21 UT and 2020 October $11 \mathrm{UT}$ lasted $6.4 \mathrm{hr}$ and $6.8 \mathrm{hr}$, respectively. Except for the use of unfiltered CCD observations early in the 
Table 2

Observations of Gault Producing Photometric Lightcurves

\begin{tabular}{|c|c|c|c|c|c|c|c|c|}
\hline Date (UTC) (1) & Telescope (2) & R.A. (3) & Decl. (4) & $r(\mathrm{au})(5)$ & $\Delta(\mathrm{au})(6)$ & $\alpha\left(^{\circ}\right)(7)$ & Filter (8) & $\theta_{s}(")(9)$ \\
\hline 2019 Jan 8 & ARC & 104815.82 & -123436.1 & 2.470 & 1.865 & 20.8 & $r^{\prime}$ & 1.3 \\
\hline 2019 Jan 18 & ARC & 104803.15 & -124242.3 & 2.451 & 1.735 & 18.8 & $r^{\prime}$ & 1.7 \\
\hline 2019 Feb 25 & ARC & 102539.71 & $\begin{array}{lll}-08 & 20 & 25.9\end{array}$ & 2.374 & 1.413 & 7.3 & $r^{\prime}$ & 1.4 \\
\hline 2019 Mar 24 & ARC & 100425.84 & -010819.5 & 2.316 & 1.406 & 12.9 & $r^{\prime}$ & 1.2 \\
\hline 2019 Apr 26 & ARC & 100229.07 & +06 1904.2 & 2.243 & 1.619 & 23.9 & $r^{\prime}$ & 2.5 \\
\hline 2019 Jun 20 & ARC & 105934.47 & +092053.9 & 2.121 & 2.150 & 27.5 & $r^{\prime}$ & 2.7 \\
\hline 2020 Jun 24 & MLO & 000626.41 & +133209.8 & 2.071 & 1.873 & 29.3 & $R$ & 2.2 \\
\hline 2020 Aug 14 & MLO & 004135.22 & +120757.8 & 2.182 & 1.416 & 21.8 & $R$ & 1.7 \\
\hline 2020 Aug 23 & MLO & 004034.98 & +103529.9 & 2.202 & 1.355 & 18.4 & $R$ & 2.2 \\
\hline 2020 Aug 23 & LOT & 004031.32 & +103232.2 & 2.202 & 1.354 & 18.3 & $R$ & 2.8 \\
\hline 2020 Aug 24 & MLO & 004019.44 & +102330.5 & 2.204 & 1.349 & 18.0 & $R$ & 1.6 \\
\hline 2020 Aug 24 & LOT & $\begin{array}{llll}00 & 40.37\end{array}$ & +101826.4 & 2.205 & 1.347 & 17.8 & $R$ & 2.5 \\
\hline 2020 Aug 27 & P200 & 003930.16 & +095027.8 & 2.210 & 1.334 & 16.9 & $r$ & 1.1 \\
\hline 2020 Sep 21 & MLO & 002406.34 & +031017.1 & 2.265 & 1.268 & 4.0 & $R$ & 2.1 \\
\hline 2020 Sep 21 & TMO & 002402.45 & +030846.4 & 2.265 & 1.268 & 3.9 & $R$ & 1.2 \\
\hline 2020 Sep 21 & LOT & 002322.57 & +025341.4 & 2.267 & 1.268 & 3.4 & $R$ & 1.7 \\
\hline 2020 Sep 21 & GIT & 002305.87 & +024737.3 & 2.268 & 1.269 & 3.2 & $r^{\prime}$ & 2.5 \\
\hline 2020 Sep 22 & TMO & 002312.10 & +024952.8 & 2.268 & 1.269 & 3.3 & $R$ & 1.6 \\
\hline 2020 Sep 22 & LOT & 002239.51 & +023747.3 & 2.269 & 1.269 & 2.9 & $R$ & 1.7 \\
\hline 2020 Sep 23 & TMO & 002223.43 & +023143.6 & 2.270 & 1.270 & 2.7 & $R$ & 1.8 \\
\hline 2020 Sep 24 & TMO & 002136.54 & +021419.8 & 2.272 & 1.271 & 2.2 & $R$ & 1.2 \\
\hline 2020 Oct 11 & MLO & $0008 \quad 16.22$ & -024428.5 & 2.310 & 1.339 & 7.5 & $R$ & 3.0 \\
\hline 2020 Oct 11 & LOT & 000802.98 & -024937.2 & 2.311 & 1.341 & 7.6 & $R$ & 2.6 \\
\hline 2020 Oct 16 & MLO & 000510.29 & -035835.4 & 2.321 & 1.373 & 9.9 & $R$ & 2.0 \\
\hline 2020 Oct 16 & GIT & 000445.91 & -04 0829.4 & 2.323 & 1.379 & 10.3 & $r^{\prime}$ & 3.1 \\
\hline 2020 Oct 20 & MLO & 000305.67 & -045151.5 & 2.330 & 1.405 & 11.7 & $R$ & 2.6 \\
\hline 2020 Oct 20 & GIT & 000243.96 & -050122.7 & 2.331 & 1.412 & 12.1 & $r^{\prime}$ & 2.7 \\
\hline
\end{tabular}

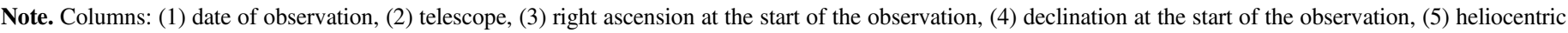

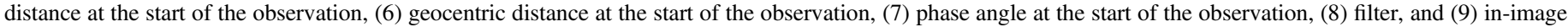
seeing at the start of the observation.

Table 3

Photometric Lightcurve Data for Figures 6 and 7

\begin{tabular}{lccc}
\hline \hline JD (1) & Mag (2) & $\sigma_{\text {Mag }}(3)$ & Observatory (4) \\
\hline 2459075.77946 & 0.000828 & 0.025020 & MLO \\
2459075.78247 & -0.012480 & 0.022338 & MLO \\
2459075.78466 & 0.005480 & 0.023636 & MLO \\
2459075.78683 & -0.001881 & 0.021923 & MLO \\
2459075.78901 & 0.013656 & 0.023636 & MLO \\
2459075.79117 & 0.002243 & 0.023137 & MLO \\
2459075.79336 & 0.002003 & 0.022937 & MLO \\
2459075.79554 & 0.007662 & 0.023037 & MLO \\
2459075.79771 & -0.002169 & 0.021340 & MLO \\
2459075.79988 & 0.007134 & 0.021839 & MLO \\
$\vdots$ & $\vdots$ & $\vdots$ & $\vdots$ \\
\hline
\end{tabular}

Note. Columns: (1) Julian date, (2) normalized magnitude, (3) normalized magnitude uncertainty, and (4) location where observations were taken.

(This table is available in its entirety in machine-readable form.)

campaign, all observations are acquired with the $R$ filter and obtained through nonsidereal tracking.

\subsection{Astrophysics Research Consortium $3.5 \mathrm{~m}$ Telescope}

Between 2019 January 8 UT and 2019 June 6 UT, we observed Gault over six campaigns with the Astrophysics Research Consortium $3.5 \mathrm{~m}$ Telescope (ARC) at the Apache Point Observatory before the asteroid headed into solar conjunction (Table 2). Individual observations created lightcurves spanning between $1 \mathrm{hr}$ (2019 March 24 UT) and 4 hr (2019 April 26 UT). Observations were taken with the ARCTIC optical CCD (Huehnerhoff et al. 2016) in the $r^{\prime}$ filter with an average seeing of 1." 8 . Throughout 2019 January and February, Gault remained visibly active, while observations between 2019 March and June displayed just a remnant tail (see Figures 1 and 5).

\subsection{Palomar Observatory 200 inch Telescope}

On 2020 August 27 UT, the Palomar Observatory 200 inch telescope (P200) observed Gault in the $r$ band. The 103 exposures each had an equivalent exposure time of $90 \mathrm{~s}$, accumulating in a total of $9270 \mathrm{~s}$ with an average seeing of $\sim 1$ ". 5 . Observations were made with the Caltech HIgh-speed Multi-color camERA (CHIMERA; Harding et al. 2016). Although CHIMERA observes in two simultaneous optical bands, we only use the $r$ band in this work.

\subsection{Zwicky Transient Facility Using the Palomar 48 inch Telescope}

Images of Gault were taken with the ZTF (Graham et al. 2019), which is mounted on the Palomar Observatory's 48 inch telescope (P48; Bellm et al. 2019; Dekany et al. 2020). ZTF images are located in the ZTF archive (Masci et al. 2019) and Gault's photometry was measured with a $5^{\prime \prime}$ radius aperture and processed using the ZChecker software (Kelley et al. 2019). The $30 \mathrm{~s}$ exposure time observations were made in the $r$ band and colorcorrected using the $g-r$ value of $0.50 \pm 0.04$ from Ye et al. (2019). Data in Figure 4 between 2018 November 1 UT and 2019 
Table 4

Observation Data for the Secular Lightcurve Taken between 2020 April 2 UT and 2020 October 14 UT by ZTF as Shown in Figure 4

\begin{tabular}{|c|c|c|c|c|c|c|c|c|}
\hline Date (UTC) (1) & R.A. (2) & Decl. (3) & $R(\mathrm{au})(4)$ & $\Delta(\mathrm{au})(5)$ & $\alpha\left(^{\circ}\right)(6)$ & mag (7) & $\chi_{a m}(8)$ & $\theta_{s}(")(9)$ \\
\hline 2020-04-02 12:14 & $21: 43: 56.3$ & $+02: 13: 35$ & 1.924 & 2.480 & 21.9 & $18.72 \pm 0.13$ & 2.525 & 4.902 \\
\hline 2020-04-02 12:21 & $21: 43: 56.9$ & $+02: 13: 38$ & 1.924 & 2.480 & 21.9 & $18.74 \pm 0.13$ & 2.399 & 5.154 \\
\hline 2020-04-02 12:27 & $21: 43: 57.4$ & $+02: 13: 41$ & 1.924 & 2.480 & 21.9 & $18.53 \pm 0.12$ & 2.286 & 3.563 \\
\hline $2020-04-02 \quad 12: 34$ & 21:43:58.0 & $+02: 13: 43$ & 1.924 & 2.480 & 21.9 & $18.93 \pm 0.21$ & 2.185 & 4.330 \\
\hline 2020-06-24 11:24 & 00:06:30.7 & $+13: 32: 24$ & 2.071 & 1.872 & 29.3 & $18.77 \pm 0.07$ & 1.273 & 1.706 \\
\hline 2020-07-08 09:03 & $00: 21: 56.5$ & $+14: 10: 18$ & 2.101 & 1.742 & 28.8 & $18.60 \pm 0.10$ & 2.066 & 2.451 \\
\hline 2020-07-09 10:56 & $00: 22: 59.0$ & $+14: 11: 41$ & 2.103 & 1.732 & 28.7 & $18.60 \pm 0.08$ & 1.262 & 2.708 \\
\hline 2020-07-10 09:17 & $00: 23: 52.1$ & $+14: 12: 40$ & 2.105 & 1.723 & 28.6 & $18.52 \pm 0.09$ & 1.81 & 1.847 \\
\hline 2020-07-17 10:05 & $00: 29: 55.6$ & $+14: 13: 52$ & 2.120 & 1.657 & 27.9 & $18.53 \pm 0.05$ & 1.331 & 1.536 \\
\hline 2020-07-19 09:20 & $00: 31: 25.3$ & $+14: 12: 06$ & 2.124 & 1.639 & 27.7 & $18.56 \pm 0.05$ & 1.515 & 1.901 \\
\hline 2020-07-20 11:26 & $00: 32: 12.3$ & $+14: 10: 43$ & 2.127 & 1.628 & 27.5 & $18.43 \pm 0.04$ & 1.11 & 1.861 \\
\hline 2020-07-20 11:30 & $00: 32: 12.4$ & $+14: 10: 43$ & 2.127 & 1.628 & 27.5 & $18.52 \pm 0.05$ & 1.105 & 1.969 \\
\hline 2020-07-23 11:03 & $00: 34: 12.6$ & $+14: 05: 21$ & 2.133 & 1.601 & 27.1 & $18.36 \pm 0.04$ & 1.125 & 1.776 \\
\hline 2020-07-25 11:56 & $00: 35: 26.9$ & $+14: 00: 20$ & 2.138 & 1.582 & 26.7 & $18.42 \pm 0.07$ & 1.076 & 1.506 \\
\hline 2020-07-26 11:06 & 00:36:00.0 & $+13: 57: 33$ & 2.140 & 1.574 & 26.6 & $18.38 \pm 0.05$ & 1.106 & 1.520 \\
\hline 2020-07-26 11:35 & 00:36:00.6 & $+13: 57: 29$ & 2.140 & 1.573 & 26.6 & $18.31 \pm 0.04$ & 1.082 & 1.534 \\
\hline 2020-07-29 11:02 & $00: 37: 33.1$ & $+13: 47: 16$ & 2.146 & 1.547 & 26.0 & $18.38 \pm 0.05$ & 1.098 & 1.763 \\
\hline 2020-08-02 08:51 & $00: 39: 12.9$ & $+13: 29: 57$ & 2.155 & 1.513 & 25.1 & $18.26 \pm 0.08$ & 1.377 & 2.476 \\
\hline 2020-08-02 09:58 & 00:39:13.8 & $+13: 29: 43$ & 2.155 & 1.512 & 25.1 & $18.19 \pm 0.06$ & 1.168 & 1.837 \\
\hline 2020-08-03 08:59 & $00: 39: 34.4$ & $+13: 24: 46$ & 2.157 & 1.504 & 24.9 & $18.20 \pm 0.08$ & 1.33 & 2.256 \\
\hline 2020-08-04 09:58 & $00: 39: 54.8$ & $+13: 19: 04$ & 2.160 & 1.495 & 24.6 & $18.17 \pm 0.09$ & 1.153 & 2.016 \\
\hline 2020-08-05 11:04 & $00: 40: 13.4$ & $+13: 13: 01$ & 2.162 & 1.486 & 24.4 & $18.21 \pm 0.07$ & 1.078 & 1.596 \\
\hline 2020-08-11 11:31 & $00: 41: 24.0$ & $+12: 31: 26$ & 2.175 & 1.438 & 22.7 & $18.15 \pm 0.04$ & 1.082 & 1.670 \\
\hline 2020-08-11 11:46 & $00: 41: 24.0$ & $+12: 31: 21$ & 2.175 & 1.437 & 22.7 & $18.09 \pm 0.04$ & 1.092 & 1.840 \\
\hline 2020-08-12 11:34 & $00: 41: 29.5$ & $+12: 23: 22$ & 2.178 & 1.430 & 22.4 & $18.10 \pm 0.04$ & 1.087 & 1.463 \\
\hline 2020-08-13 10:19 & $00: 41: 33.2$ & $+12: 15: 26$ & 2.180 & 1.423 & 22.0 & $18.05 \pm 0.24$ & 1.085 & 1.361 \\
\hline 2020-08-14 11:06 & $00: 41: 35.1$ & $+12: 06: 27$ & 2.182 & 1.415 & 21.7 & $18.12 \pm 0.04$ & 1.077 & 1.436 \\
\hline 2020-08-14 11:39 & $00: 41: 35.1$ & $+12: 06: 15$ & 2.182 & 1.415 & 21.7 & $18.07 \pm 0.04$ & 1.096 & 1.521 \\
\hline 2020-08-15 09:49 & $00: 41: 35.4$ & $+11: 57: 55$ & 2.184 & 1.408 & 21.4 & $18.24 \pm 0.16$ & 1.104 & 1.612 \\
\hline 2020-08-18 09:37 & $00: 41: 25.2$ & $+11: 28: 55$ & 2.191 & 1.387 & 20.3 & $18.00 \pm 0.04$ & 1.104 & 1.912 \\
\hline 2020-08-20 09:38 & 00:41:09.4 & $+11: 07: 50$ & 2.195 & 1.373 & 19.6 & $17.95 \pm 0.04$ & 1.096 & 2.111 \\
\hline $2020-08-20 \quad 10: 05$ & $00: 41: 09.2$ & $+11: 07: 38$ & 2.195 & 1.373 & 19.6 & $17.93 \pm 0.04$ & 1.078 & 1.776 \\
\hline 2020-08-23 11:39 & $00: 40: 31.0$ & $+10: 32: 42$ & 2.202 & 1.354 & 18.3 & $17.86 \pm 0.04$ & 1.143 & 1.828 \\
\hline 2020-08-25 08:49 & 00:39:59.9 & $+10: 09: 44$ & 2.206 & 1.342 & 17.5 & $17.92 \pm 0.04$ & 1.131 & 1.716 \\
\hline 2020-08-26 09:03 & $00: 39: 40.5$ & $+09: 56: 56$ & 2.209 & 1.337 & 17.1 & $17.86 \pm 0.05$ & 1.107 & 2.218 \\
\hline 2020-08-27 09:29 & $00: 39: 19.3$ & $+09: 43: 41$ & 2.211 & 1.331 & 16.7 & $17.88 \pm 0.04$ & 1.082 & 1.510 \\
\hline 2020-09-02 09:11 & $00: 36: 41.5$ & $+08: 19: 24$ & 2.224 & 1.303 & 13.9 & $17.76 \pm 0.11$ & 1.079 & 1.499 \\
\hline 2020-09-02 09:32 & $00: 36: 41.0$ & $+08: 19: 10$ & 2.224 & 1.303 & 13.8 & $17.65 \pm 0.11$ & 1.142 & 1.998 \\
\hline 2020-09-06 08:04 & $00: 34: 28.6$ & $+07: 18: 12$ & 2.233 & 1.288 & 11.9 & $17.64 \pm 0.06$ & 1.179 & 1.839 \\
\hline 2020-09-12 09:28 & $00: 30: 27.4$ & $+05: 37: 19$ & 2.247 & 1.273 & 8.6 & $17.49 \pm 0.05$ & 1.162 & 1.998 \\
\hline 2020-09-12 10:21 & $00: 30: 25.8$ & $+05: 36: 41$ & 2.247 & 1.273 & 8.6 & $17.53 \pm 0.05$ & 1.248 & 1.954 \\
\hline 2020-09-18 08:41 & $00: 25: 56.9$ & $+03: 51: 55$ & 2.260 & 1.267 & 5.3 & $17.35 \pm 0.03$ & 1.144 & 2.073 \\
\hline 2020-09-20 09:39 & $00: 24: 19.3$ & $+03: 15: 05$ & 2.264 & 1.268 & 4.1 & $17.30 \pm 0.03$ & 1.226 & 1.548 \\
\hline 2020-09-21 07:17 & $00: 23: 36.0$ & $+02: 58: 44$ & 2.266 & 1.268 & 3.6 & $17.26 \pm 0.03$ & 1.164 & 1.785 \\
\hline 2020-09-21 07:34 & $00: 23: 35.4$ & $+02: 58: 32$ & 2.266 & 1.268 & 3.6 & $17.27 \pm 0.03$ & 1.15 & 2.031 \\
\hline 2020-09-23 07:54 & $00: 21: 57.3$ & $+02: 21: 58$ & 2.271 & 1.270 & 2.4 & $17.17 \pm 0.03$ & 1.139 & 1.996 \\
\hline 2020-09-26 06:20 & $00: 19: 33.9$ & $+01: 28: 48$ & 2.277 & 1.275 & 0.8 & $17.07 \pm 0.03$ & 1.22 & 1.520 \\
\hline 2020-09-27 09:09 & $00: 18: 39.1$ & $+01: 08: 41$ & 2.280 & 1.278 & 0.4 & $16.96 \pm 0.03$ & 1.22 & 1.612 \\
\hline 2020-10-04 07:07 & $00: 13: 10.5$ & $-00: 52: 54$ & 2.295 & 1.302 & 3.8 & $17.39 \pm 0.05$ & 1.233 & 1.894 \\
\hline 2020-10-06 06:03 & $00: 11: 42.3$ & $-01: 25: 55$ & 2.300 & 1.311 & 4.9 & $17.42 \pm 0.05$ & 1.265 & 1.621 \\
\hline 2020-10-08 06:07 & $00: 10: 14.9$ & $-01: 58: 53$ & 2.304 & 1.321 & 6.0 & $17.48 \pm 0.03$ & 1.252 & 1.853 \\
\hline 2020-10-12 07:07 & 00:07:29.7 & $-03: 02: 29$ & 2.313 & 1.346 & 8.0 & $17.62 \pm 0.04$ & 1.252 & 2.267 \\
\hline 2020-10-14 07:09 & 00:06:14.4 & $-03: 32: 21$ & 2.317 & 1.360 & 9.0 & $17.67 \pm 0.03$ & 1.262 & 1.848 \\
\hline
\end{tabular}

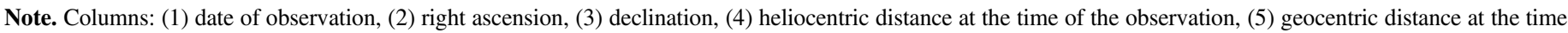
of the observation, (6) phase angle at the time of the observation, (7) magnitude, (8) airmass of observation, and (9) in-image seeing.

(This table is available in machine-readable form.)

February 10 UT are adapted from Ye et al. (2019). A full list of the ZTF observations starting on 2020 April 2 UT and ending on 2020 October 14 UT is located in Table 4. The seeing varied between 1 ." 5 and 2 !. 5 , and the airmasses varied from 1.4 to 2.6 during the span of our observations.

\subsection{GROWTH-India Telescope}

We observed Gault on multiple nights with the $0.7 \mathrm{~m}$ GROWTH-India telescope (GIT) using the SDSS $r^{\prime}$ filter and an Apogee KAF3200EB camera giving a $\sim 11^{\prime} \times 7$ '.5 

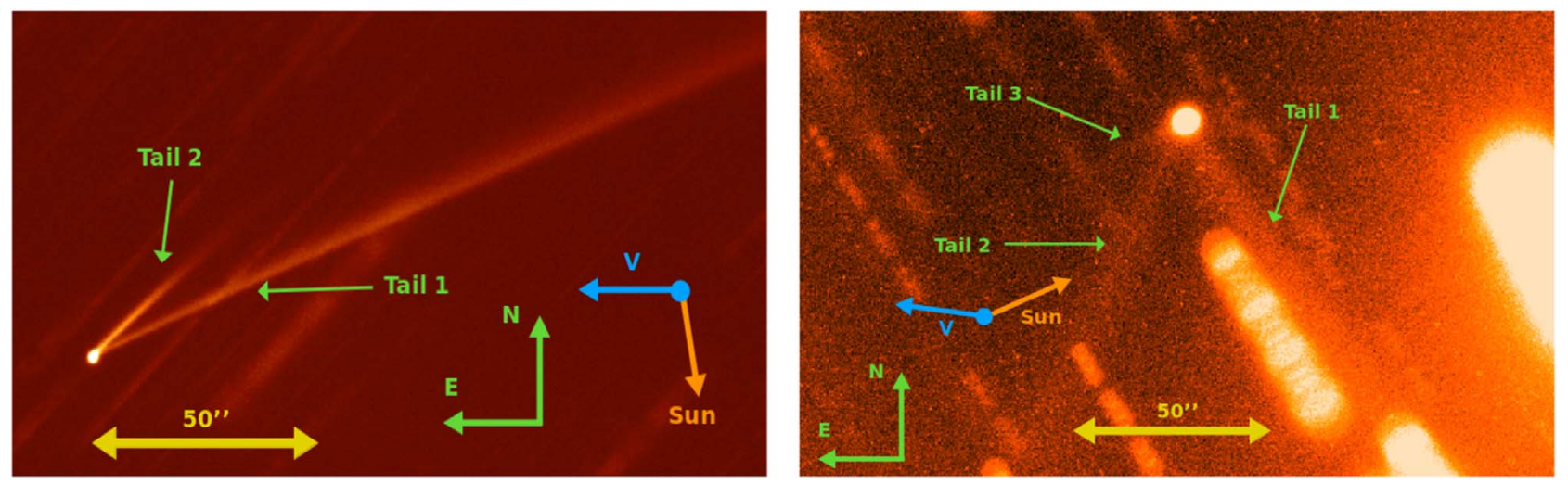

Figure 1. Left: $4680 \mathrm{~s}$ deep-stack image of Gault from the ARC $3.5 \mathrm{~m}$ telescope at the Apache Point Observatory on 2019 February 25 UT. The two tails indicate two separate epochs of activity. Right: $4320 \mathrm{~s}$ deep-stack image of Gault from the ARC $3.5 \mathrm{~m}$ on 2019 April 26 UT after it had produced a third tail.

field of view. Due to the slow motion of Gault, we used sidereal tracking and took multiple exposures. Data were acquired in $120 \mathrm{~s}$ exposures on 2020 September 21, followed by $180 \mathrm{~s}$ exposures on 2020 October 16 and 20. Data were downloaded and processed in real time at our data processing machine at IIT Bombay. We calibrated images for processing by applying bias correction and flat-fielding, obtained an astrometry solution using the offline engine of astrometry.net (Lang et al. 2010), and finally removed cosmic rays via AstroSCRAPPY (McCully \& Tewes 2019) package. Photometry was performed using a PyRAF-based processing pipeline. We cross-matched the sextractor-identified (Bertin 2011) sources in the GIT image with \# II/349/ps1 catalog (Flewelling 2018) using vizier. Magnitudes were calibrated by correcting for zero points.

\subsection{GROWTH Coordinated Observations}

On 2020 September 21 UT, LOT, GIT, MLO, and the Table Mountain Observatory (TMO) $1.0 \mathrm{~m}$ telescope participated in a $20 \mathrm{hr}$ relay of observing Gault for photometric lightcurve variation. MLO started the relay on 2020 September 21 04:24:47 UT (airmass 2.0) and ran continuous observations of 120 s exposure times until 11:35:27 UT (airmass 2.0) the same day, totaling 22,800 s of exposure time. LOT took over shortly after at 14:26:07 (airmass 1.2) amassing 14,130 $\mathrm{s}$ of $90 \mathrm{~s}$ exposures before finishing at 20:29:09 UT (airmass 2.5). GIT observed Gault for roughly $6 \mathrm{hr}$ in 135 images, amassing 16,200 s of exposure starting from 17:21:31 UT (airmass 1.2), in the middle of the LOT observations, and ending at 23:18:07 UT (airmass 2.5). TMO observed Gault for roughly $4 \mathrm{hr}$ in 225 images amassing 13,500 $\mathrm{s}$ of exposure starting at 06:36:37 UT (airmass 1.3), in the middle of MLO's observations. Typical seeing for GIT is $2 . " 5$ and for TMO is $1 . " 5$. We were able to take data with the Table Mountain Observatory despite the degraded conditions caused by a nearby wildfire. Additional coordination among the GROWTH network includes MLOLOT observations on 2020 August 23 and 24 UT and 2020 October 11 UT, TMO-LOT on 2020 September 22 UT, and MLO-GIT on 2020 October 16 and 20 UT.

\section{Results}

\subsection{Active and Inactive States of Gault}

Follow-up observations with the ARC $3.5 \mathrm{~m}$ telescope at the Apache Point Observatory on 2019 February 25 UT showed

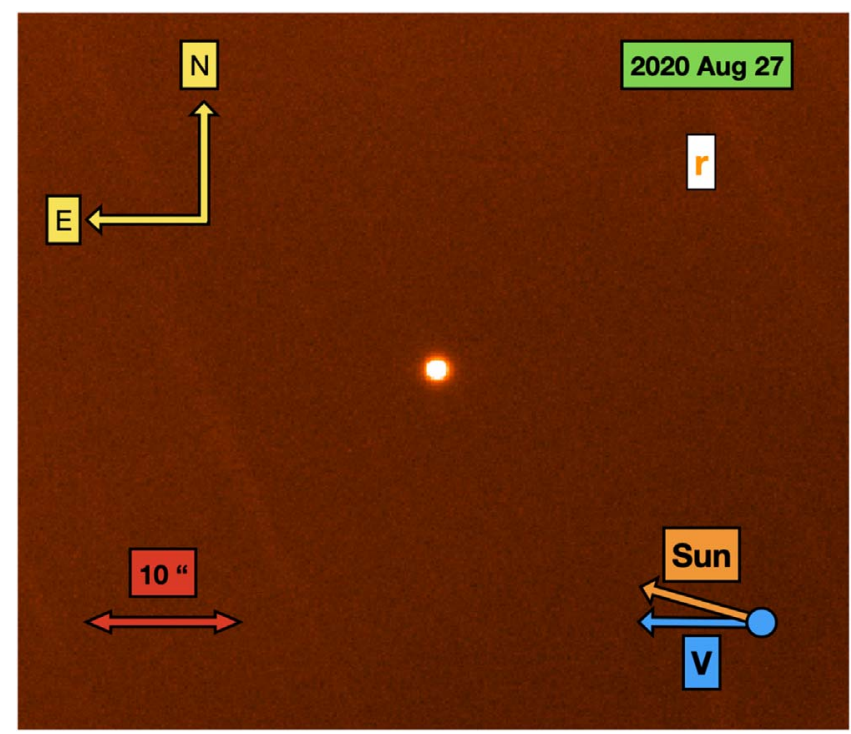

Figure 2. Deep-stack image of Gault taken with the P200 telescope in $10390 \mathrm{~s}$ images culminating in $9270 \mathrm{~s}$ on 2020 August 27 UT in the $r$ band. The image displays a lack of coma or southwest-facing tail, implying the inactivity of Gault.

evidence for multiple tails. A deep-stack image consisting of $4680 \mathrm{~s}$ of exposure is shown on the left side of Figure 1. The surface brightness of Gault in this stack is 24.0 mag $\operatorname{arcsec}^{-2}$ within a $10,000 \mathrm{~km}$ radius aperture. The right side of Figure 1 shows Gault's third tail in a $4320 \mathrm{~s}$ deep-stack image from the ARC $3.5 \mathrm{~m}$ telescope on 2019 April $26 \mathrm{UT}$, and the 10,000 km surface brightness was calculated to be $23.8 \mathrm{mag} \mathrm{arcsec}^{-2}$.

The images were combined in deep, median stacks centered on Gault and then used to compute the calibrated $r$-band photometry from comparisons to similar deep-stacked images of reference stars with solar colors from the same initial image. We referenced photometry of the reference star from the PanSTARRS catalog (Chambers et al. 2016).

Both surface brightnesses were brighter than the surface brightness of $25.8 \mathrm{mag} \operatorname{arcsec}^{-2}$ found when it was inactive in 2020 June by Purdum et al. (2020). Our deep-stack image taken by P200 in the $r$ band is shown in Figure 2, which lacks cometary features and has a surface brightness of $26.3 \mathrm{mag} \mathrm{arcsec}^{-2}$, also dimmer than the surface brightnesses from early 2019 . 


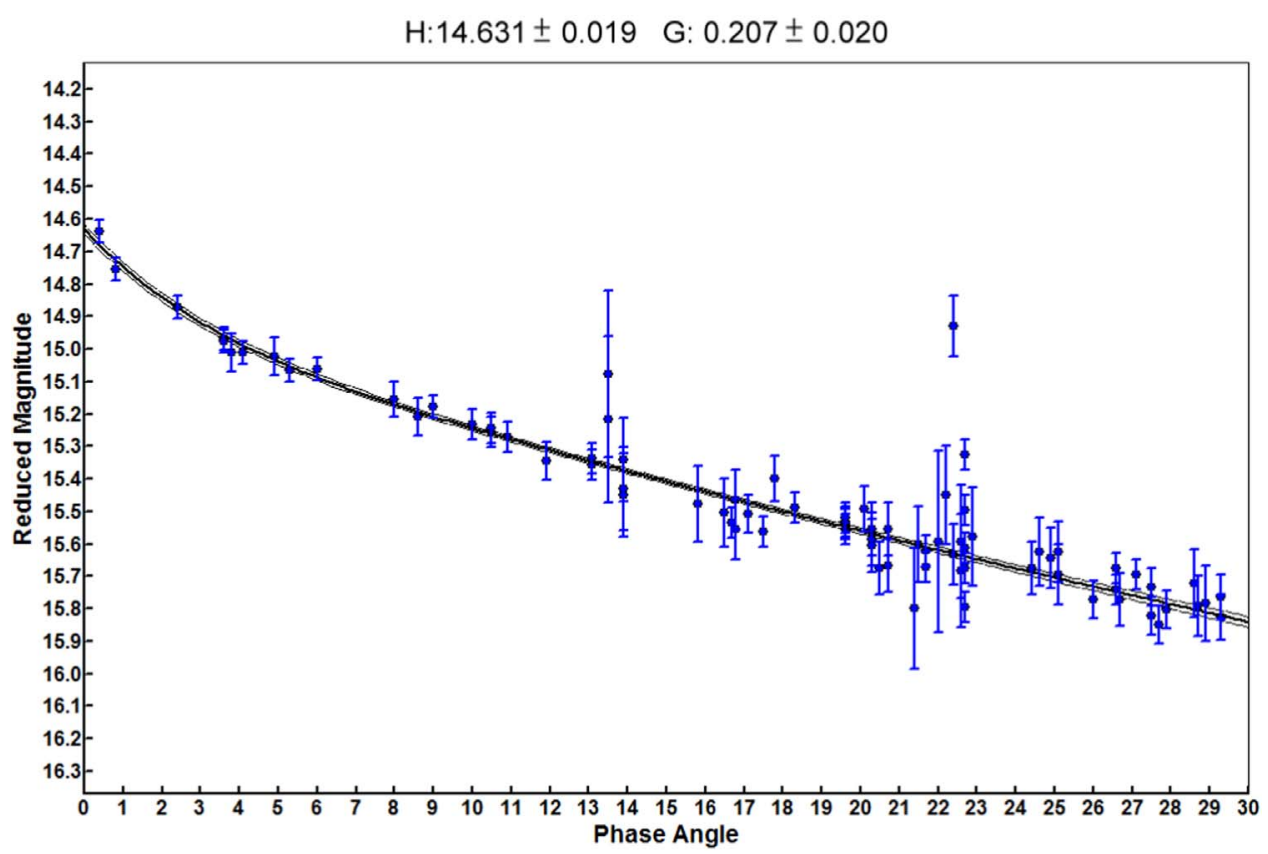

Figure 3. Reduced magnitude of Gault from Equation (1) as a function of phase angle. The data points are taken from P48 observations with ZTF starting 2020 April 2 UT and ending 2020 October 14 UT. The line of best fit is based on $r-5 \log _{10}(R \Delta)$ in Equation (1) using $H_{r}=14.631 \pm 0.019$ and $G=0.207 \pm 0.020$.

\subsection{Secular Photometry and Updated Absolute Magnitude}

Figure 3 shows Gault's reduced-magnitude phase curve from ZTF data taken between 2020 April 2 UT to 2020 October 14 UT. The reduced-magnitude data is described by $r-5 \log _{10}(R \Delta)$, where $R$ and $\Delta$ are the heliocentric and geocentric distances. We can then find the $r$-band absolute magnitude $H_{r}$ and phase-slope parameter $G$ by fitting the reduced magnitudes to the phase function of the form

$$
r-5 \log _{10}(R \Delta)=H-2.5 \log _{10}\left[(1-G) \Phi_{1}(\alpha)+G \Phi_{2}(\alpha)\right.
$$

where $r$ is the $r$-band magnitude of Gault taken with a $5^{\prime \prime}$ radius aperture, $\alpha$ is the phase angle of the asteroid at the time of midexposure, and $\Phi_{1}$ and $\Phi_{2}$ are two basis functions normalized at unity for $\alpha=0^{\circ}$ (Bowell et al. 1989; Muinonen et al. 2010; Pravec et al. 2012).

These best-fit parameters are $H_{r}=14.631 \pm 0.019$ and $G_{r}=0.207 \pm 0.020$ after the asteroid had exited solar conjunction and was no longer active. This is significantly fainter than the value of $14.31 \pm 0.01$ measured by ZTF when Gault was last seen to be inactive in 2017 (Ye et al. 2019). This could be caused by the change in observing geometry over time, creating a different line-of-site projection of Gault's light-scattering cross section. The lack of a large lightcurve amplitude for Gault discussed below implies that the detection of Gault at the limiting magnitude of the survey at the limits of the phase curve was due to the viewing geometry of Gault rather than rotational variations in its brightness (Jedicke et al. 2016). Using the updated predicted absolute magnitude of Gault with the blue line in Figure 4, we can see the instability in the brightness of Gault over time using $5^{\prime \prime}$ radius aperture photometry.

Figure 4 shows the time-series $r$-band ZTF photometry between 2020 June and October. Gault exited solar conjunction and was observed between 2020 April 2 UT $(M J D=58941)$ and 2020 October 14 UT (MJD = 59136). Comparing the measured equivalent $r$-band magnitude of Gault from photometry measured in ZTF observations taken on these dates with the predicted magnitude of Gault based on our measured $r$-band absolute magnitude of $H_{r}=14.631 \pm 0.019$ and phase function slope value $G=0.207 \pm 0.020$, we do not see any significant brightening in the actual magnitude of Gault compared to the predicted magnitudes. This is in contrast to the brightness of Gault in the "active" portion of its lightcurve between 2018 November 1 UT $(\mathrm{MJD}=58423)$ and 2019 February 10 UT (MJD = 58524). Between 2019 February 24 UT (MJD = 58538) and 2019 July 9 UT (MJD $=58673)$, the measured brightness and predicted brightness begin to become similar, suggesting that the enhanced cross section of Gault caused by dust within its vicinity was beginning to diminish in contrast to the increase in brightness of comets whose brightness are observed to increase with steadily increasing activity as they approach the Sun (Bolin et al. 2020b, 2021).

\subsection{Time-series Lightcurves}

While Gault was active in 2019, the ARC $3.5 \mathrm{~m}$ telescope at Apache Point Observatory took short-period lightcurve images, as shown in Figure 5. Much like Figure 6 in Jewitt et al. (2019b), this plot shows little variation in the lightcurve while the asteroid was experiencing activity and producing cometlike features. The variations that do occur in these lightcurves have small-amplitude peaks and are caused by noise consistent with the uncertainty values in their individual differential photometry.

The flatness of the lightcurves is noted even as the brightness of Gault began to resemble its predicted brightness based on its pre-activity $H_{r}$ seen in Figure 4 . In order to determine the rotational period of Gault after it returned to an inactive state, we obtained coordinated long-term lightcurves on nine separate dates starting in 2020 August, with the longest single lightcurve of $19 \mathrm{hr}$ on 2020 September 21 UT. The results are displayed in Figures 6 and 7. 


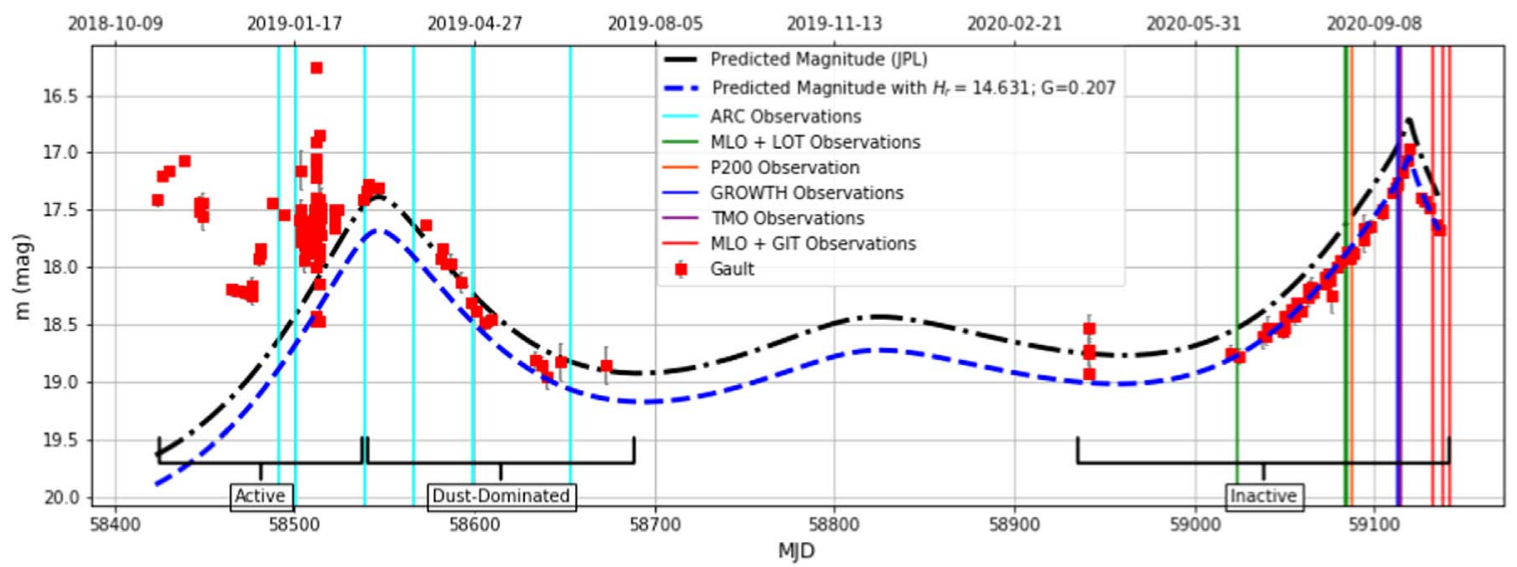

Figure 4. Secular photometry of Gault from 2018 November 1 UT $(M J D=58423)$ to 2020 October 14 UT (MJD = 59136). The red points are the apparent magnitudes of Gault taken by the Palomar Observatory 48 inch telescope and ZTF in the $r$ band over this time span. Data presented from before 2019 February 10 UTC are adapted from Ye et al. (2019). The black line is the predicted apparent $V$-band magnitude from JPL HORIZON's ephemeris service. The blue line indicates what the predicted magnitude should be given new values for the absolute magnitude $H=14.631 \pm 0.019$ and slope parameter $G=0.207 \pm 0.020$. While the asteroid is active, the data do not line up with the predicted magnitude due to dust obscuring the surface of the asteroid. As Gault continued to be dust dominated as it entered solar conjunction before MJD 58700, the predicted and observed magnitudes started to align again. After exiting solar conjunction, Gault shows that it aligns with the new predicted values during inactivity. The vertical lines indicate the observation epochs in this work.

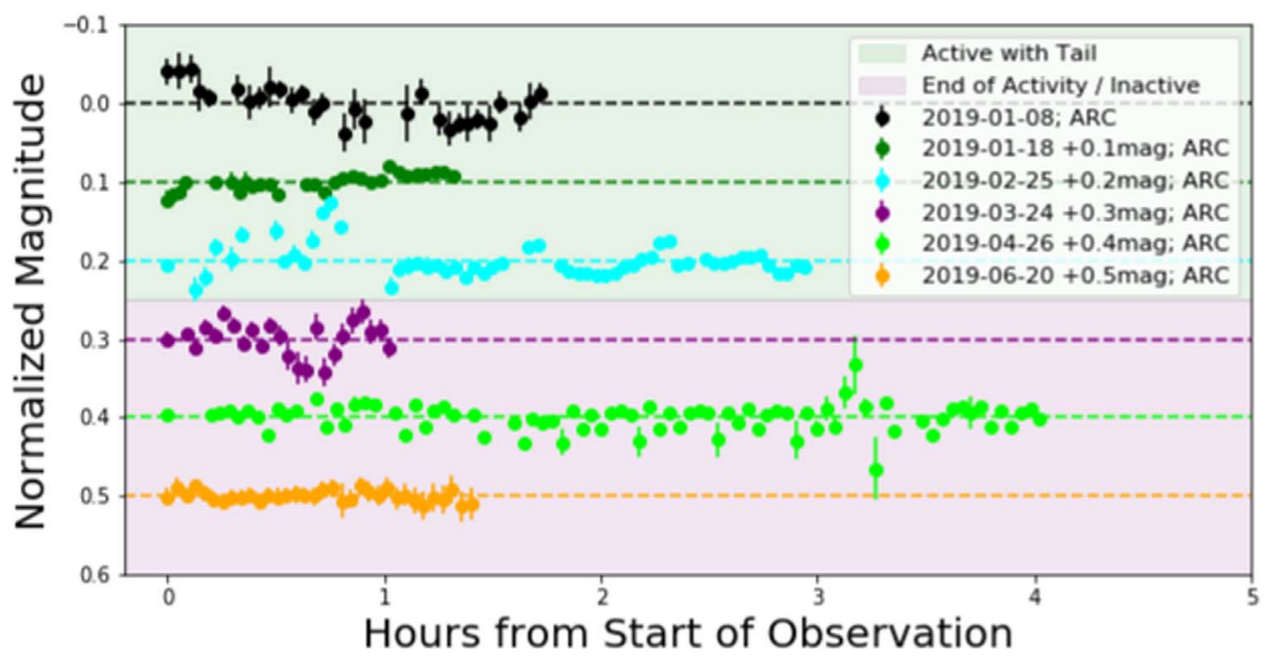

Figure 5. 2019 data from the ARC $3.5 \mathrm{~m}$ telescope. The data are organized chronologically from top to bottom and are offset by $0.1 \mathrm{mag}$ from each other. The first three dates correspond to the epochs in which Gault was active and where the ZTF photometry is brighter than predicted, and the last three dates were obtained during the dust-dominated epochs that followed, as seen in Figure 4.

The separate observatories are indicated by color, with MLO as red, LOT as green, P200 as orange, TMO as purple, and GIT as blue. Each lightcurve for each observatory has an additional reference star lightcurve normalized to -0.3 mag to show that the reference stars used to calculate the differential photometry of Gault did not vary over time (or if so, not at the same period and amplitude as the asteroid's lightcurves). The reference star lightcurves have small error bars and have not been corrected for airmass or weather effects on their photometry and therefore are curved, but display no strong signs of periodicity.

Each date in Figures 6 and 7 also contains arrows to indicate the small-amplitude, periodic peaks in the asteroid lightcurves based on multiples of $2.5 \mathrm{hr}$ intervals from each other. Solidcolored arrows denote the primary peaks that represent a single rotation of the asteroid, while white arrows point to the half-period peaks at multiples of $1.25 \mathrm{hr}$ from the primary peaks. Due to the minuscule 0.1 mag amplitude of the peaks, many of them are overcome by the noise in the photometry and therefore only a handful appear on each date. Figure 8 shows an example of phasefolded lightcurves from the GROWTH relay of observations on
2020 September 21 UT. Similar to Figures 6 and 7, the telescopes are color-coordinated, and their lightcurves are folded by the double-peaked $2.5 \mathrm{hr}$ period. The MLO and LOT data are stacked in the bottom plot and display modest primary and secondary peaks caused by Gault's periodic rotation. Additional phasefolded lightcurves of each observation date in Figures 6 and 7 can be found in the Appendix.

A Lomb-Scargle (Lomb 1976) periodogram was constructed from MLO and LOT data of Gault ranging from 2020 August to October and is shown along with a folded lightcurve of Gault in Figure 9. The differential photometry technique we used to create the periodogram relied on determining the difference between the brightness of Gault and the comparison stars in the same field of view to acquire Gault's lightcurve. The comparison stars we selected depended on the maximum frame width that we used through one run and on the similarity of the FWHM estimated between Gault and the chosen stars. By comparing the reference stars' lightcurves, some variable stars were ruled out in the photometric analysis. To combine several photometry runs through different nights, the mean 

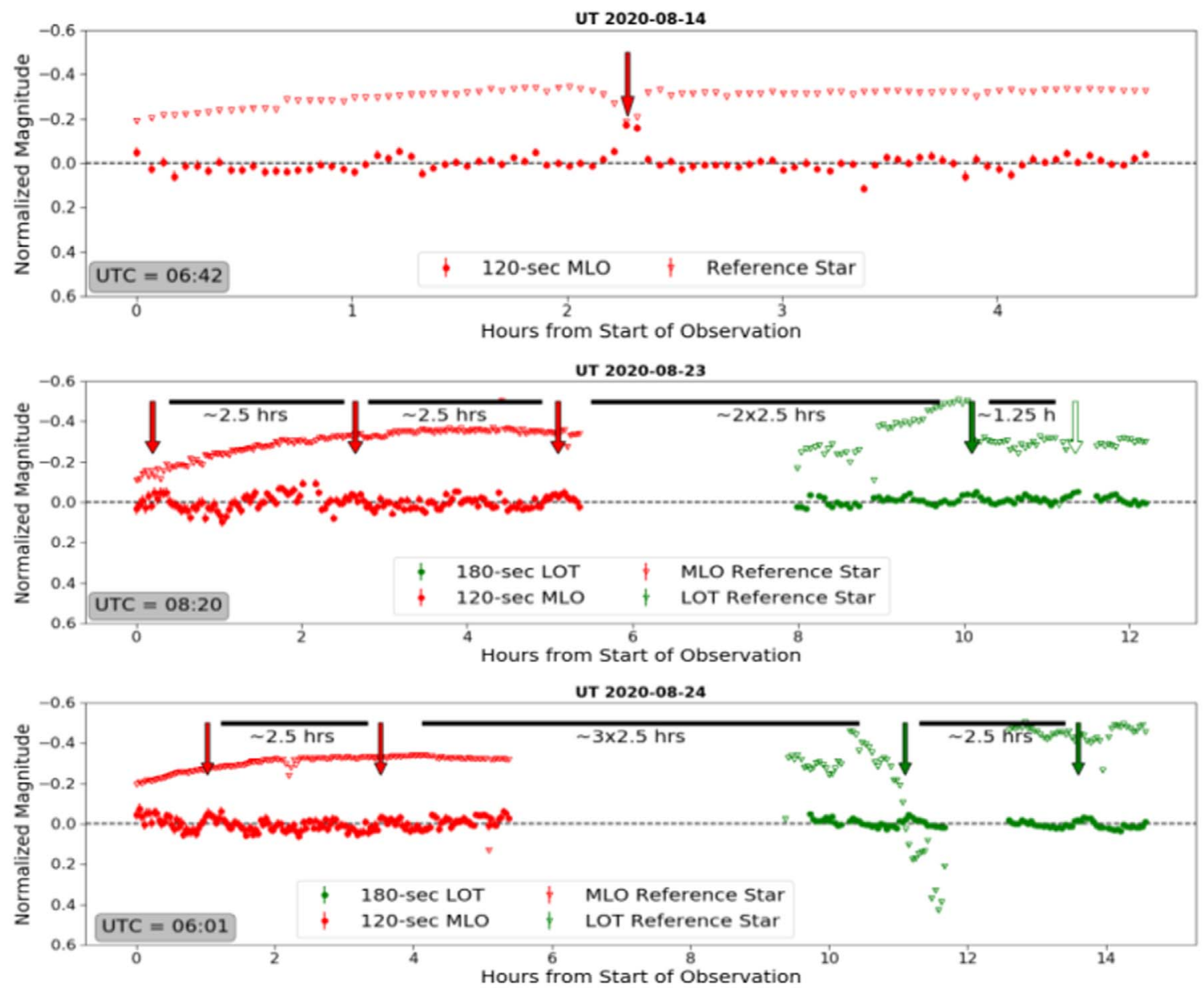

UTC 2020-08-27
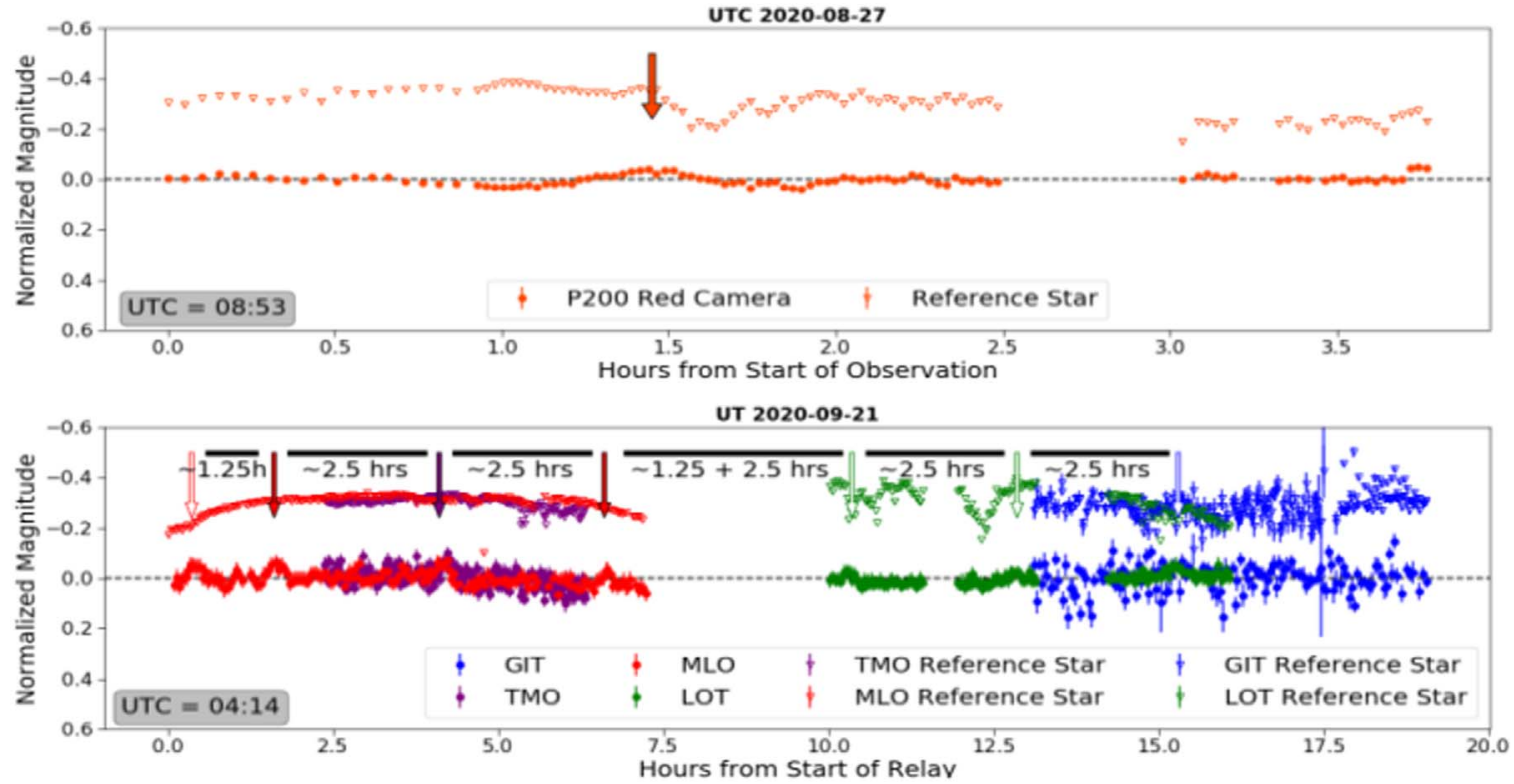

Figure 6. Coordinated long-term photometric lightcurves of Gault and various reference stars (offset $-0.3 \mathrm{mag}$ ) starting from 2020 August 14 UT and organized chronologically to 2020 September 21 UT. The observatories are color-coordinated, and the bumps in the lightcurve caused by the rotation of $\sim 2.5$ hr are denoted by arrows. The primary peaks are marked by solid arrows while secondary half-period peaks are denoted with white arrows. The lightcurves continue through 2020 October 20 UT in Figure 7.

values of each run were automatically scaled using an IDL routine that we created. We then searched for significant periodicities using the Lomb-Scargle periodogram functions on the combined lightcurve data to find the most likely rotation period of Gault. The frequency analysis from the strong peak near $\sim 20$ cycles day $^{-1}$ in the left panel of Figure 9 gives a rotation period of $\sim 1.25 \mathrm{hr}$, which corresponds to a singlepeaked lightcurve. It is natural to assume a double-peaked 


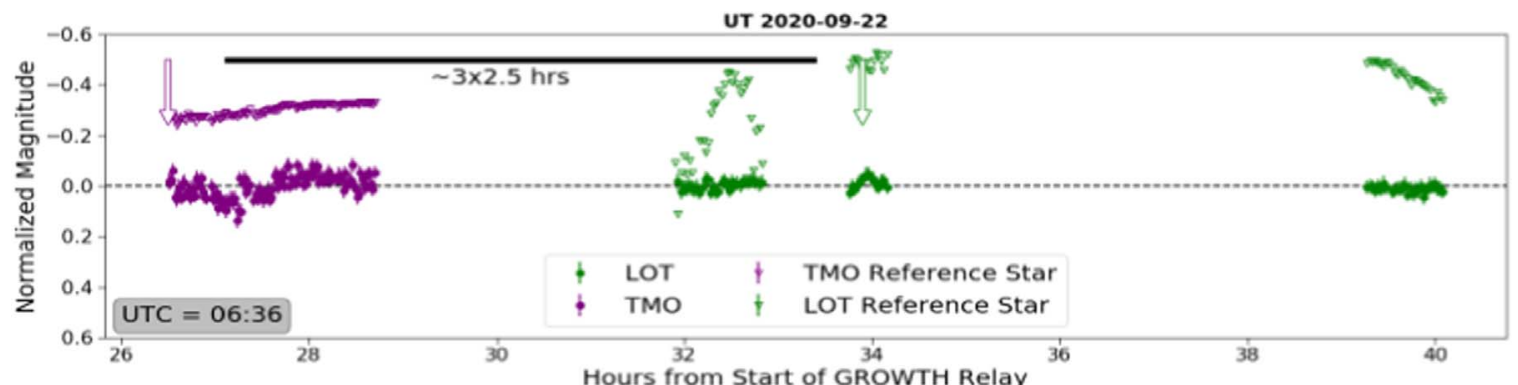

UT 2020-09-23
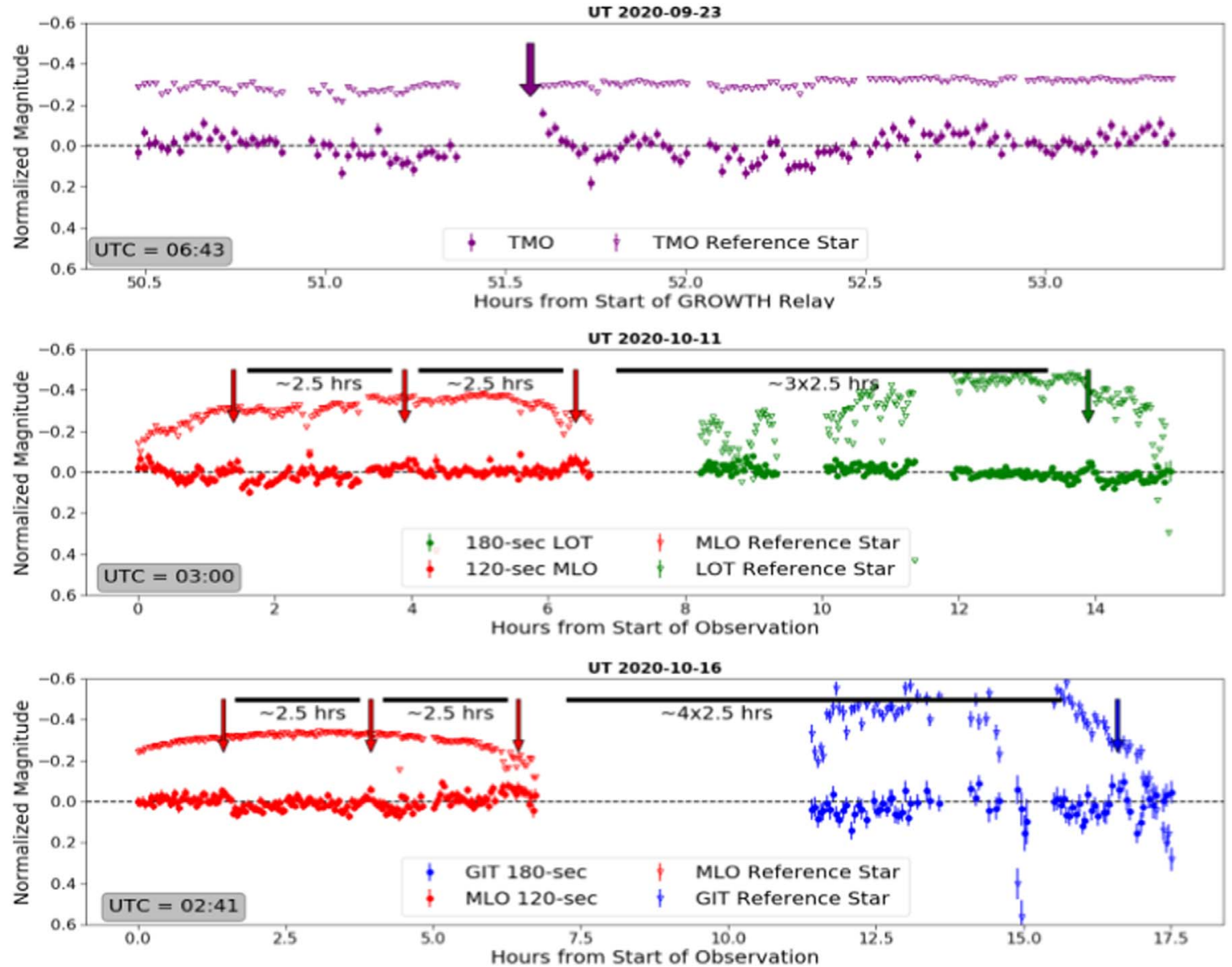

UT 2020-10-20

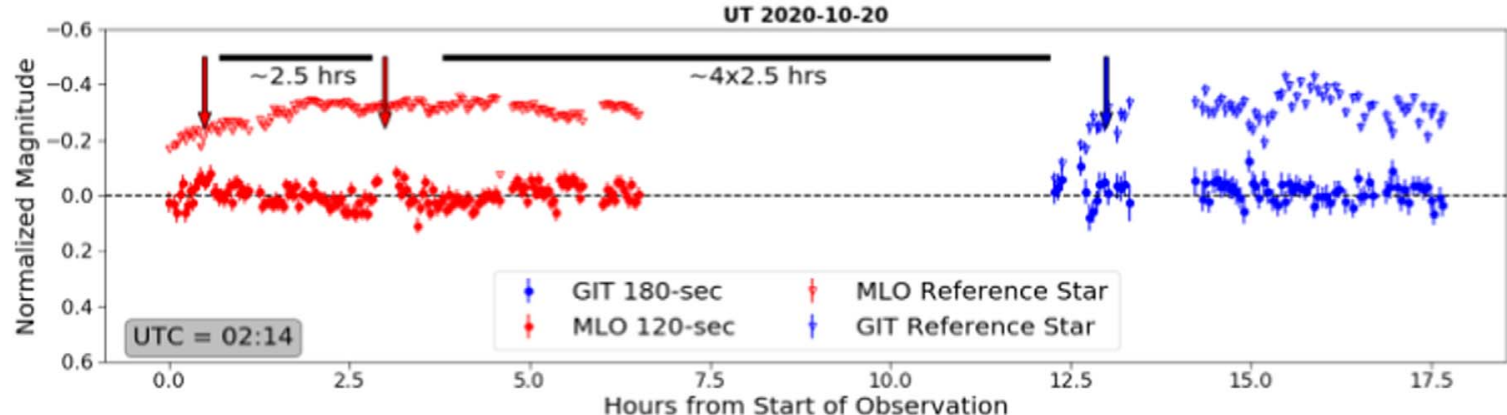

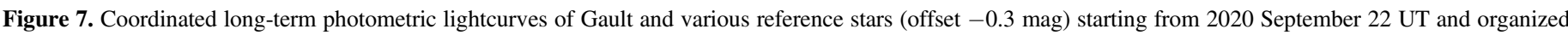

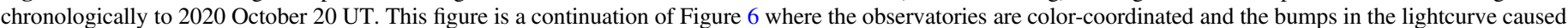

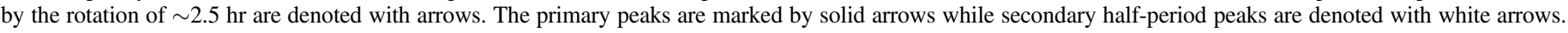

lightcurve for Gault, which computes to a sidereal rotation period of $2.49 \pm 0.07 \mathrm{hr}$. The right panel of Figure 9 displays the lightcurve folded by the rotation period of $2.49 \mathrm{hr}$, which corresponds to a double-peaked lightcurve. The uncertainty here of $\pm 0.07 \mathrm{hr}$ is estimated using the bootstrap method
(Press et al. 1986), which removed $\sqrt{N}$ data points from the time-series lightcurves and recalculated the period value from the Lomb-Scargle periodogram. This process was repeated 10,000 separate times with the resulting central value of $2.49 \mathrm{hr}$ and a $1-\sigma$ uncertainty estimate of $0.07 \mathrm{hr}$. 


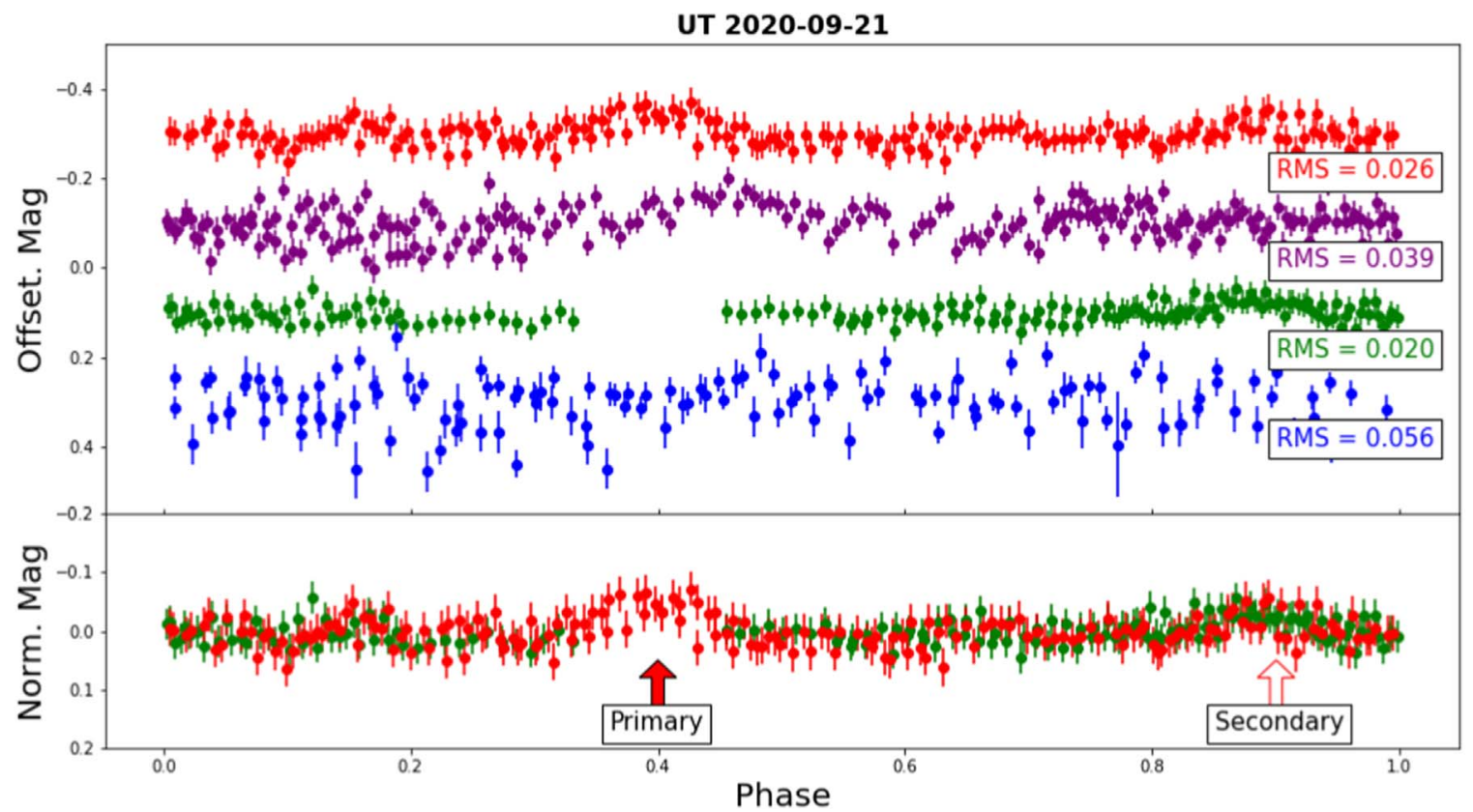

Figure 8. Phase-folded lightcurve of Gault on 2020 September 21 UT from the GROWTH relay of observations. Each telescope's lightcurve was folded by 2.5 hr, and the MLO and LOT data were stacked to display the primary and secondary peaks of the double-peaked lightcurve. The rms value is shown for each lightcurve as a reference for the amplitude of the noise in the data.
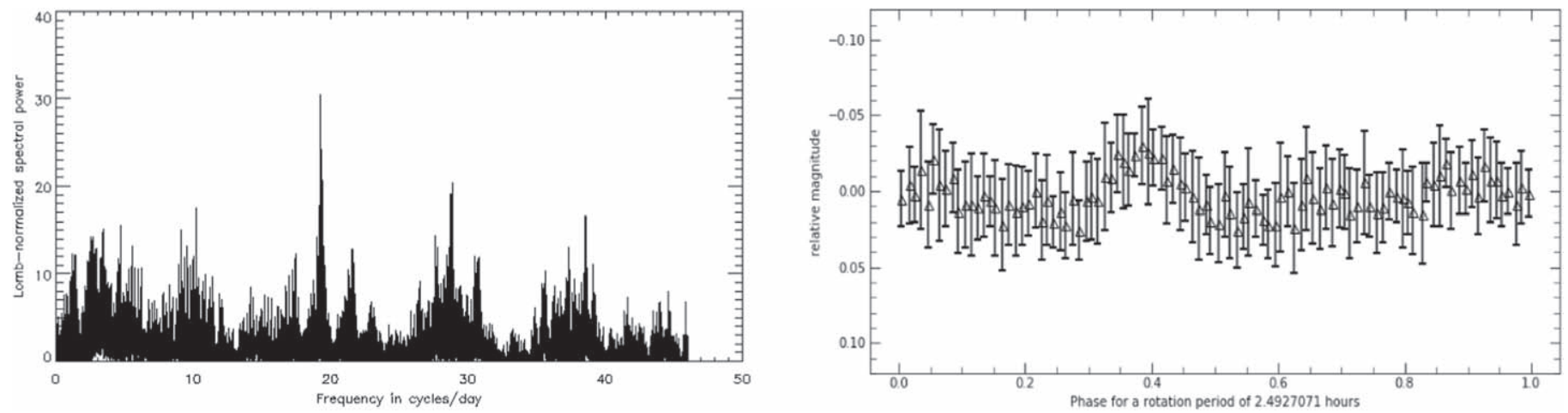

Figure 9. Left: Lomb-Scargle periodogram of Gault's lightcurve data from MLO and LOT observations starting 2020 August 23 UT and ending 2020 October 20 UT. Right: folded lightcurve of Gault from MLO and LOT with a period of $2.5 \mathrm{hr}$ averaged over 10 individual MLO and LOT lightcurves taken over six nights of data taken between 2020 August 23 UT and ending 2020 October 20 UT.

The amplitudes of the MLO and LOT lightcurves are low, so it is somewhat difficult to recognize a continuous variation in brightness in the folded version of the lightcurve, as seen on the right in Figure 9. Here, the folded phase curves averaged by rebinning them in phase space with a bin size of 0.01 and coadded into an average, and the error bars are the $1 \sigma$ scatter in data per phase bin.

\section{Discussion and Summary}

The surface brightness profiles taken from deep-stacked images of Gault from the MLO $1.0 \mathrm{~m}$ on 2020 July 21 UT (Purdum et al. 2020) and P200 on 2020 August 27 UT indicate that Gault is no longer active after it appeared to have an outburst of material that caused multiple tails to form starting in 2018 October (Ye et al. 2019). The surface brightness measurements of $25.8 \mathrm{mag} \mathrm{arcsec}^{-2}$ and $26.3 \mathrm{mag} \mathrm{arcsec}^{-2}$, from MLO and Palomar, respectively, are fainter compared to the surface brightness values of Gault from the ARC $3.5 \mathrm{~m}$ Telescope on 2019 February 9 UT, had a measurement of $24 \mathrm{mag} \mathrm{arcsec}-2$ (Purdum et al. 2020). The fainter measurements in 2020 could mean that Gault no longer has material surrounding it and can be deemed inactive.

Gault's deactivation can also be seen over time in the photometry from ZTF observations of Gault in Figure 4. The activity of Gault is apparent on the left side of the plot, with the data being much brighter and more variable than the predicted magnitudes from JPL's HORIZONS ephemeris service. ${ }^{26}$ The outbursts for Gault's first two tails were estimated to have occurred on 2018 October $18 \pm 5$ UT and 2018 December $24 \pm 1$ UT (Ye et al. 2019), which is during the "active" portion of the lightcurve (see Figure 4). The third tail was much dimmer than the first two and so the exact time of initiation is uncertain, but Jewitt et al. (2019b) estimate it to be 2019 February $10 \pm 7$ UT, right at the time the "active" portion of Figure 4 ends.

After Gault had produced its third tail, the ZTF photometry started to line back up with the predicted magnitudes from JPL's HORIZONS ephemeris service, indicating that the active

\footnotetext{
${ }^{26}$ https://ssd.jpl.nasa.gov/?horizons
} 
stage had come to an end. However, this alignment did not last as the photometry from ZTF started to dip below the predicted magnitudes in Figure 4. The misalignment occurred while Gault no longer had tails but was still surrounded by dust, which can skew photometric measurements. The right side of Figure 4, however, shows that Gault's photometry exiting solar conjunction was more stable than the active stage of the plot, therefore providing more evidence for Gault's inactivity.

Interestingly, Gault no longer aligned with the predicted magnitudes from HORIZONS ephemeris service after it had exited solar conjunction. We found that altering the phase parameter $G$ in Equation (1) from 0.25 to 0.21 and the absolute magnitude $H$ from JPL's HORIZONS' 14.3 to 14.6 realigns the photometry in Figure 4. This phenomenon can be attributed to the observing geometry of Gault changing throughout its orbit. The brighter absolute magnitude $H_{r}$ during the ARC observations in 2019 (see Figure 4) could be due to pole-on observations, while observations during different viewing geometries would result in smaller absolute magnitudes in 2020. The JPL HORIZONS' ephemeris service shows the estimated ecliptic longitude of Gault during observations by $\mathrm{Ye}$ et al. (2019) in 2017 November was $\sim 58^{\circ}$ while our observations range from $\sim 3^{\circ}$ to $\sim 5^{\circ}$.

Photometric lightcurve observations with the ARC $3.5 \mathrm{~m}$ while Gault was still dust dominated (see Figure 5) in 2019 started to show some variation as the activity on Gault diminished, but the low amplitude of the variations was not enough to conclude a rotation period. Our observations of Gault in 2020 also produced low-amplitude lightcurves (see Figures 6 and 7), even though it no longer displayed signs of activity. The viewing geometry is also much different for our observations in 2020 than from when it was active in 2019. This means that Gault could have a spherical or top-shaped geometry like the near-Earth asteroids Ryugu and Bennu (Hirabayashi et al. 2020). It is also worth noting that the nearEarth Asteroid (3200) Phaethon was imaged by Arecibo radar observations and was found to have a round, top-like shape when it passed by Earth in 2017 December (Taylor et al. 2019). At that time, Kim et al. (2018) found Phaethon's peak amplitude was small, $\sim 0.1 \mathrm{mag}$, which is somewhat similar to our data in Figures 6 and 7.

Despite the low amplitude in the lightcurves, we did notice periodic small-amplitude peaks and found they are separated by roughly $2.5 \mathrm{hr}$ and placed arrows in Figures 6 and 7 to indicate the estimations. Some secondary peaks occurred at $1.25 \mathrm{hr}$ intervals from the primary peaks due to the asteroid's geometry displaying a double-peaked lightcurve. Several peaks in the Gault lightcurves show little to no variation in the reference star lightcurve (see August 24, September 21, 23, October 20 in Figures 6 and 7), which is an indication that the peaks are nonanomalous and are a result of the rotation of the asteroid. The periodogram in Figure 9 shows Gault has a rotation period of $2.49 \pm 0.07 \mathrm{hr}$, assuming the frequency of $\sim 20$ cycles day $^{-1}$ $(\sim 1.25 \mathrm{hr})$ corresponds to a double-peaked lightcurve. Figures 8 and 11-19 in the Appendix show the lightcurves for our observations spanning 2020 August 14 UT to 2020 October 20 UT (see Figures 6 and 7) folded by a period of 2.5 $\mathrm{hr}$. The stronger primary peak and a fainter secondary peak of Gault's double-peak lightcurve are denoted with arrows, and each figure shows the lightcurves stacked by date. An rms calculation was made for each phase-folded lightcurve as a reference for the noise amplitude when looking at the primary

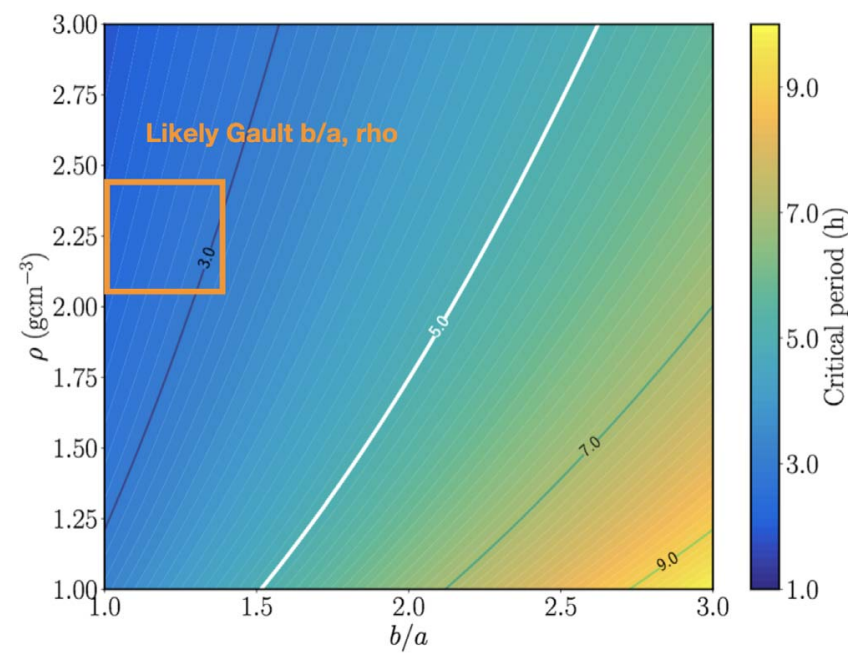

Figure 10. Adapted from Bolin et al. (2018). The critical rotation period of an asteroid based on the axial ratio $b / a$ and the density $\rho$. The likely critical period for Gault is indicated in the orange box, roughly around 2.0-3.0 hr.

and secondary bumps. Some dates show the peaks clearly, while others do not, due to observation quality. Because the single-peak period of $1.25 \mathrm{hr}$ from our Lomb-Scargle periodogram is physical for an asteroid of Gault's geometry, we only display the double-peak phase-folded lightcurves.

With the assumption that Gault is nearly spherical or top shaped (see Harris et al. 2014 on the determination of an asteroid's shape from its lightcurve), we assume the $b / a$ axial ratio is close to $1-1.3$ and $b / c \sim 1.3$ given its maximum possible lightcurve amplitude of $\sim 0.1-0.3$, as seen in our data and the data from Kleyna et al. $(2019)^{27}$ and the relation between $b / a$ and lightcurve amplitude of $b / a=10^{0.4 \Delta M}$ from Binzel et al. (1989). It should be noted that the axial ratio inferred from the observed lightcurve amplitude can be affected by the angle between the spin pole and the observer (Vokrouhlický et al. 2017; Hanuš et al. 2018); however, the consistently small lightcurve amplitude from different viewing geometries in the 2019 and 2020 apparitions seems to favor a smaller axial ratio. The critical breakup period of a strengthless ellipsoid as a function of axial ratio is given by Jewitt et al. (2017a) as

$$
P_{\text {crit }}=\left(\frac{b}{a}\right)\left[\frac{3 \pi}{G \rho}\right]^{1 / 2},
$$

where $\rho$ is the density of the ellipsoid and $G$ is the Newtonian gravitational constant. Gault should have a density of roughly equal to $2.2 \mathrm{~g} \mathrm{~cm}^{-3}$ (Marsset et al. 2019; Sanchez et al. 2019), consistent with other S-type asteroids (Carry 2012). Figure 10 presents the critical period with which Gault would start shedding surface material as a function of the axial ratio and density. An orange box labeled in Figure 10 is likely to contain the critical rotation period for an object with Gault's geometry. A rotation period like the one we have found at $2.5 \mathrm{hr}$ seems to be at or near the critical period of Gault and therefore could be the cause of the activity started in 2018. Previous authors have also proposed Gault's activity was caused by rotational

\footnotetext{
27 Although Kleyna et al. (2019) show a small-amplitude magnitude variation similar to our results, it is worth noting that their results were found while Gault was showing signs of activity.
} 
instability induced from the YORP effect (Ferrín et al. 2019; Jewitt et al. 2019b; Kleyna et al. 2019). Jewitt et al. (2015) determined that the YORP spin-up timescale for Gault should be roughly $22 \mathrm{Myr}$, much shorter than the $100 \mathrm{Myr}$ timescale for the reorientation of the spin of a $\sim 4 \mathrm{~km}$ asteroid by nondestructive collisions (Farinella et al. 1998). It is therefore possible that Gault may experience rotational fission if it continues to be spun up past its rotational breakup limit (e.g., Jewitt et al. 2017b; Moreno et al. 2017). Scheeres (2015) theorizes that the surface of an asteroid after YORP spin-up can be "perched" and ready for failure in multiple areas, which can cause multiple epochs of activity similar to active asteroid P/ 2013 P5 (Jewitt et al. 2013; Hainaut et al. 2014). We believe Gault could have had multiple epochs of activity after an initial YORP spin-up despite losing angular momentum from ejected particles.

Some asteroids are observed to be spinning beyond their rotational limit, but this is typically for asteroids smaller than the kilometer scale (Pravec et al. 2008) to smaller than the meter scale (Bolin et al. 2014, 2020a), which are held together by cohesive forces (Sánchez \& Scheeres 2014). However, it should be noted that examples of kilometer-scale asteroids have been found rotating faster than their critical period (Chang et al. 2017) from fast-rotating-asteroid searches in wide-field optical surveys (Chang et al. 2016, 2019).

Other causes for activity are somewhat less likely than rotational instability. For instance, unlike main asteroid belt comets (Jewitt et al. 2015), there are many clues that point away from ice sublimation as the driver for Gault's activity. Because Gault is $S$ type and a member of the inner main belt Phocaea family, it likely formed inside the snow line and therefore would not experience sublimation (Vernazza \& Beck 2016). Additionally, Gault's activity was observed with the ARC $3.5 \mathrm{~m}$ telescope when Gault was located at a heliocentric distance of 2.41 au on 2019 February 9 UT. When Gault was observed to be inactive by the MLO $1.0 \mathrm{~m}$ telescope on 2020 June 24 UT, it had a heliocentric distance of 2.07 au, suggesting the shorter distance to perihelion did not drive the activity. Collision events are also unlikely to be the cause for activity due to the multiple epochs of activity occurring in the few months Gault was active. Thermal disintegration is also somewhat unlikely due to Gault's low-eccentricity orbit. Active asteroids with this kind of driver typically have highly eccentric orbits, which cause large changes in temperature that lead to fracture (Delbo et al. 2014; Jewitt et al. 2015). Other processes such as solar radiation pressure are unlikely due to Gault's rotation constantly changing the orientation with respect to the Sun. Therefore, we find that Gault's activity was likely driven by rapid rotation at its critical period of $2.5 \mathrm{hr}$.

Based on observations obtained with the Samuel Oschin Telescope 48 inch and the 60 inch Telescope at the Palomar Observatory as part of the Zwicky Transient Facility project. ZTF is supported by the National Science Foundation under grant No. AST-2034437 and a collaboration including Caltech, IPAC, the Weizmann Institute for Science, the Oskar Klein Center at Stockholm University, the University of Maryland,
Deutsches Elektronen-Synchrotron and Humboldt University, the TANGO Consortium of Taiwan, the University of Wisconsin at Milwaukee, Trinity College Dublin, Lawrence Livermore National Laboratories, and IN2P3, France. Operations are conducted by COO, IPAC, and UW.

This work was supported by the GROWTH project funded by the National Science Foundation under PIRE grant No. 1545949.

Part of this work was performed under the auspices of the US Department of Energy by Lawrence Livermore National Laboratory under Contract DE-AC52-07NA27344.

B.T.B. and F.J.M. acknowledge support from NASA with grant No. 80NSSC19K0780.

C.F. gratefully acknowledges the support of his research by the Heising-Simons Foundation (\# 2018-0907).

M.C. acknowledges support from the National Science Foundation with grant No. PHY-2010970.

This publication has made use of data collected at Lulin Observatory, partly supported by MoST grant 108-2112-M008-001.

C.C.N. thanks the funding from MOST grant 104-2923-M008-004-MY5.

C.Z. acknowledges support from JPL's internal research funds of the R\&TD, JROC, and ESD HBCU/MSI programs.

J.N.P. and R.Q. acknowledge support from JPL's ESD HBCU/MSI program under subcontract 1659249.

The work of J.H. has been supported by the Czech Science Foundation through grant 20-08218S and by the Charles University Research program No. UNCE/SCI/023.

V.B., K.S., and H.K. thank Kunal Deshmukh for help with data processing. The GROWTH-India telescope is a $70 \mathrm{~cm}$ telescope with a 0.7 degree field of view, set up by the Indian Institute of Astrophysics and the Indian Institute of Technology Bombay with support from the Indo-US Science and Technology Forum (IUSSTF) and the Science and Engineering Research Board (SERB) of the Department of Science and Technology (DST), Government of India (https://sites.google. com/view/growthindia/). It is located at the Indian Astronomical Observatory (Hanle), operated by the Indian Institute of Astrophysics (IIA). The GROWTH-India project is supported by SERB and administered by IUSSTF.

H.K. thanks the LSSTC Data Science Fellowship Program, which is funded by LSSTC, NSF Cybertraining grant \#1829740, the Brinson Foundation, and the Moore Foundation; his participation in the program has benefited this work.

Facilities: Apache Point Astrophysical Research Consortium $3.5 \mathrm{~m}$ telescope, GROWTH-India Telescope, Lulin Optical Telescope, Mount Laguna Observatory 40 inch Telescope, P48 Oschin Schmidt telescope/Zwicky Transient Facility, Table Mountain Observatory.

Software: Astropy (Astropy Collaboration et al. 2013, 2018), ZChecker (Kelley et al. 2019), Aperture Photometry Tool (Laher et al. 2012).

\section{Appendix}

Figures 11-19 below display additional phase-folded lightcurves of Gault based on the lightcurves in Figures 6 and 7. 


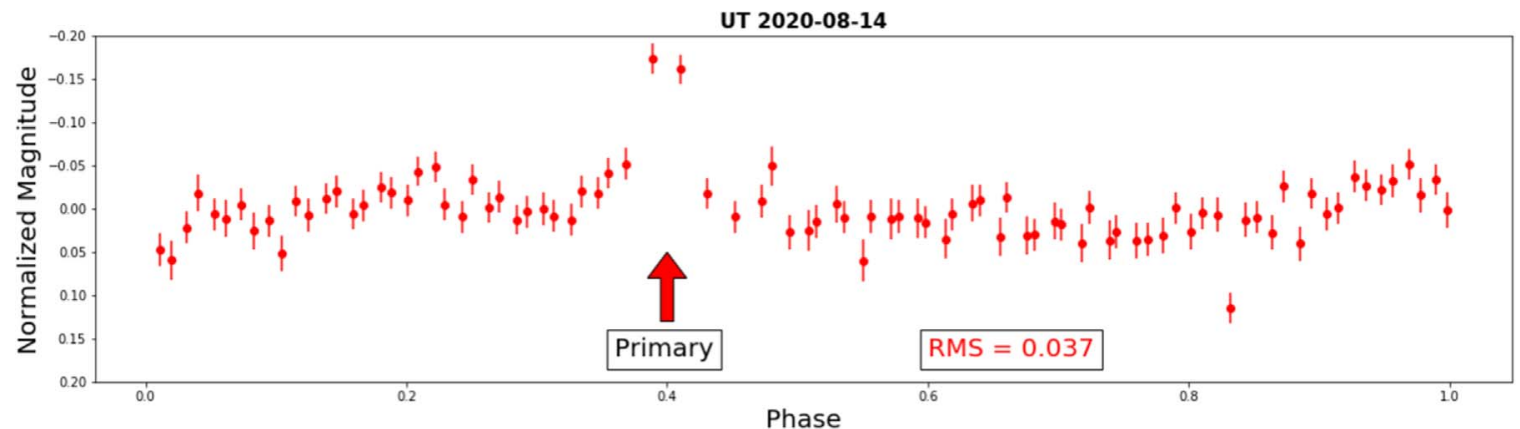

Figure 11. Phase-folded lightcurve of Gault on 2020 August 14 UT from MLO folded by $2.5 \mathrm{hr}$. Unfortunately, Gault passed over a star in the same observation frame and caused the magnitude to spike more than expected just as it was showing a peak. The secondary peak is somewhat undefined compared to the noise.

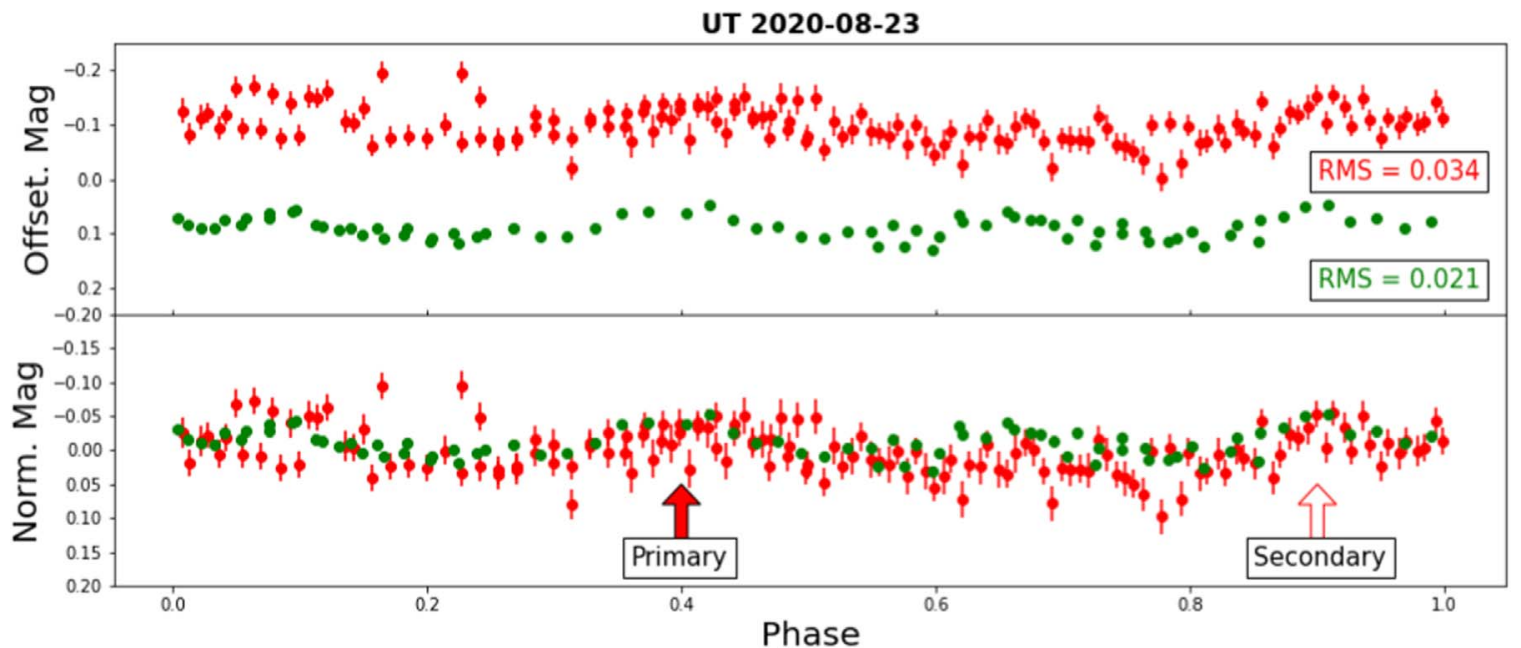

Figure 12. Phase-folded lightcurve of Gault on 2020 August 23 UT from MLO and LOT folded by 2.5 hr. Although the primary peak is not well defined in the MLO observations, the LOT observations show a promising bump. The secondary peak also is somewhat defined in both lightcurves.

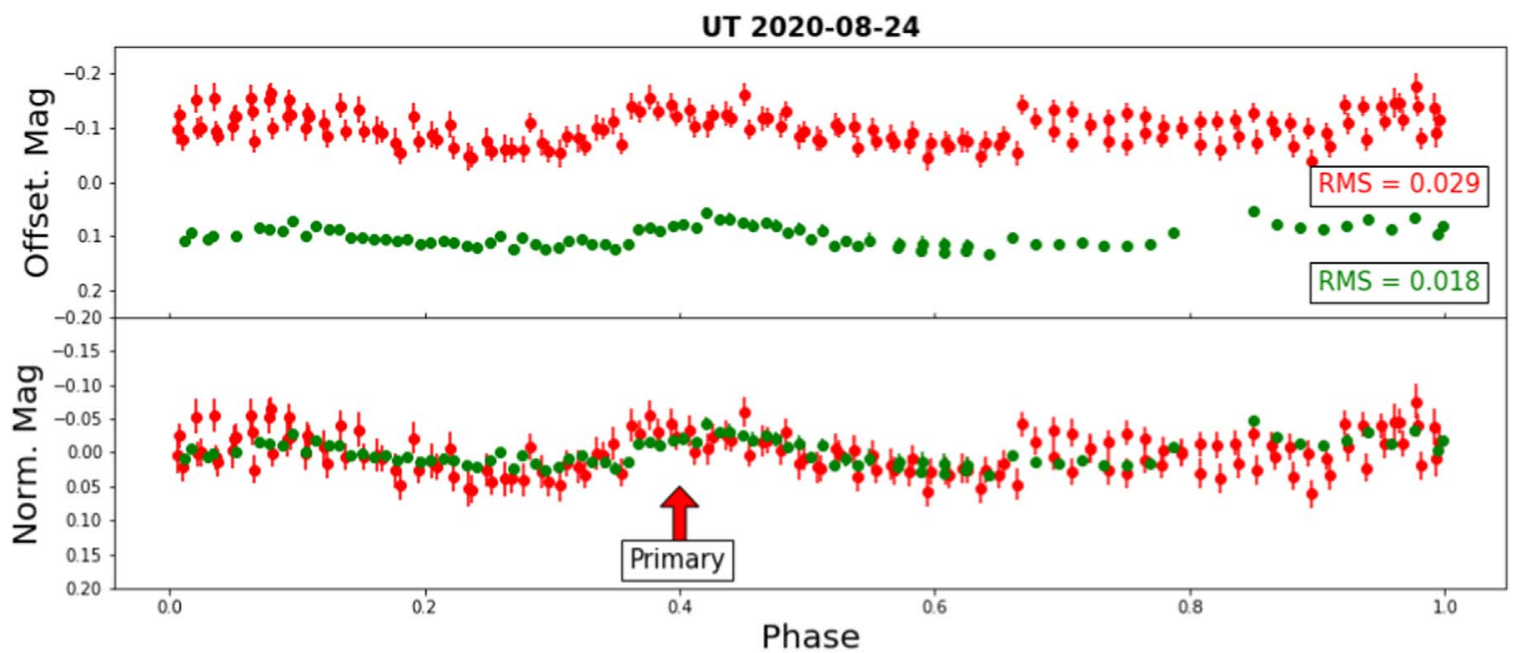

Figure 13. Phase-folded lightcurve of Gault on 2020 August 24 UT from MLO and LOT folded by $2.5 \mathrm{hr}$. Both lightcurves display a promising primary bump but neither have a convincing secondary bump. 


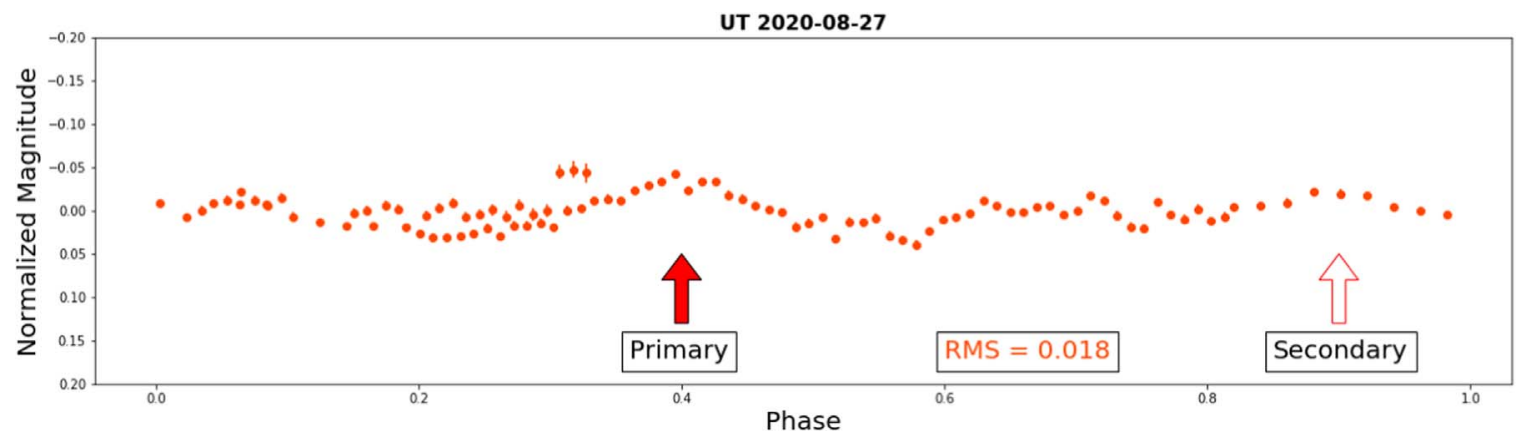

Figure 14. Phase-folded lightcurve of Gault on 2020 August 27 UT from P200 folded by $2.5 \mathrm{hr}$. Although this lightcurve is short, the high-quality observation displays a strong primary peak and convincing secondary peak.

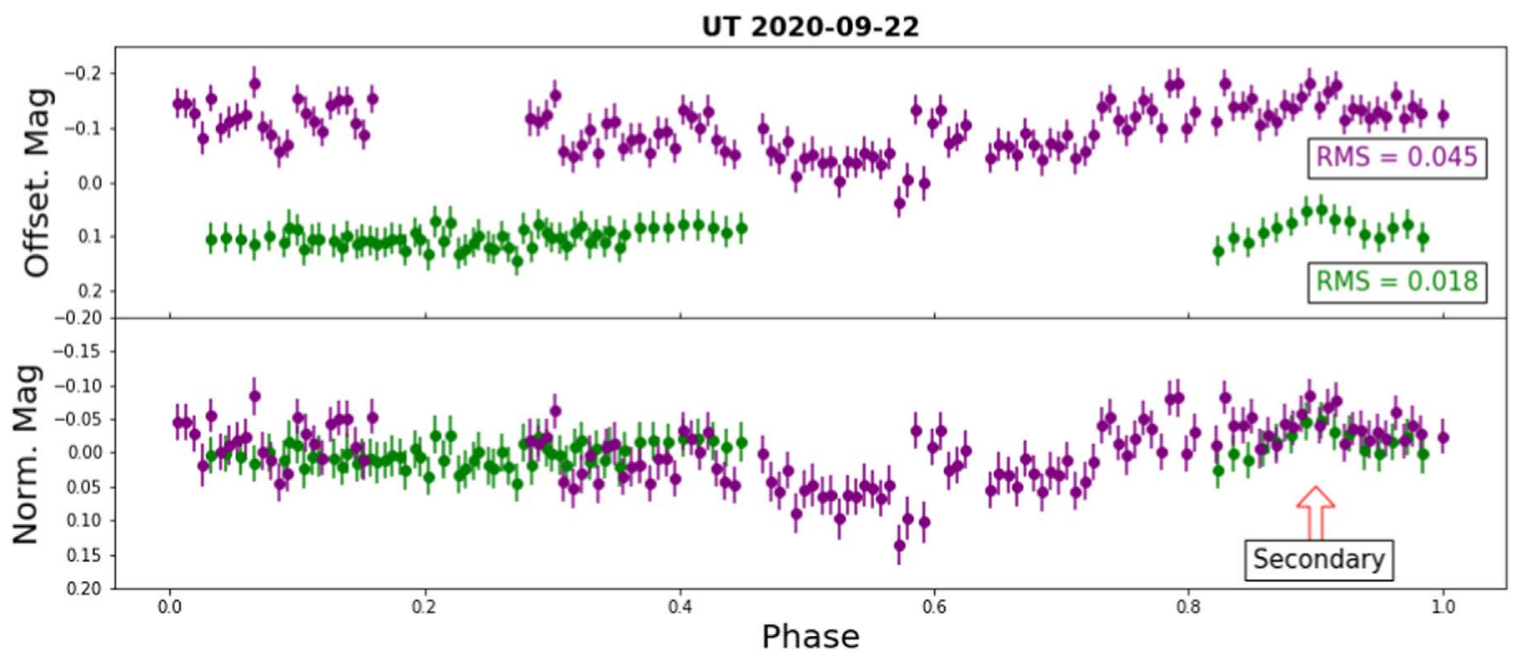

Figure 15. Phase-folded lightcurve of Gault on 2020 September 22 UT from LOT and TMO folded by 2.5 hr. The primary peak does not rise beyond the noise in either observation, but both observations display a promising secondary peak. (In this case, it is important to note that the primary and secondary peaks are both separated in time by $2.5 \mathrm{hr}$ from other primary and secondary peaks in the lightcurve, so therefore one could arbitrarily say that the primary is strong and the secondary is weak for this date.)

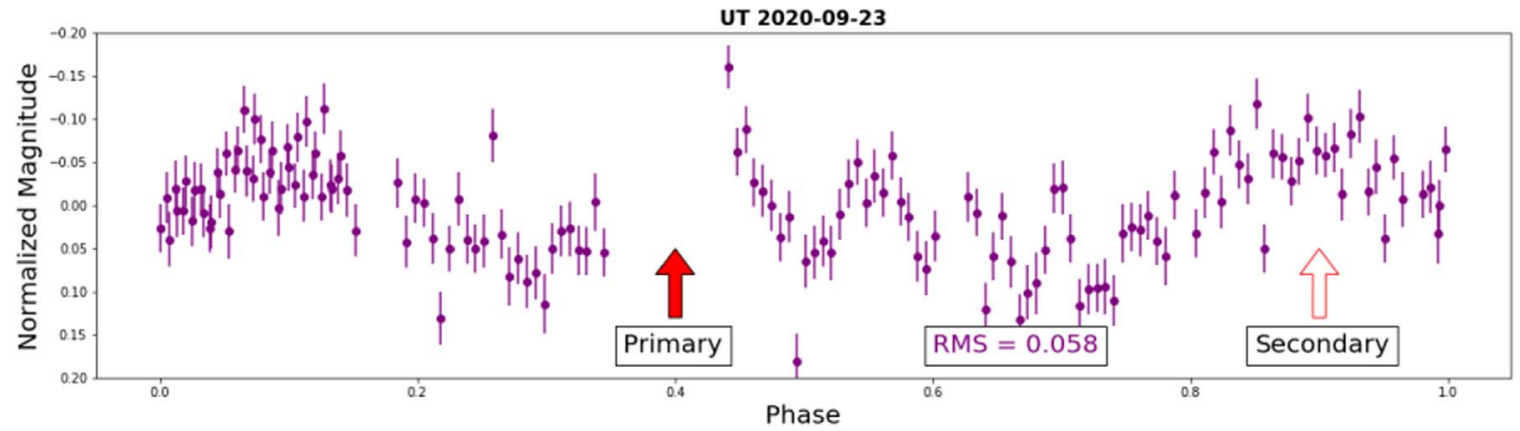

Figure 16. Phase-folded lightcurve of Gault on 2020 September 23 UT from TMO folded by $2.5 \mathrm{hr}$. Observations from TMO during 2020 September were affected by a local wildfire, and therefore, the noise in the lightcurves from TMO is abnormally high. The primary looks to have occurred just as observations were temporarily halted but were resumed in time to see a modest secondary peak. 


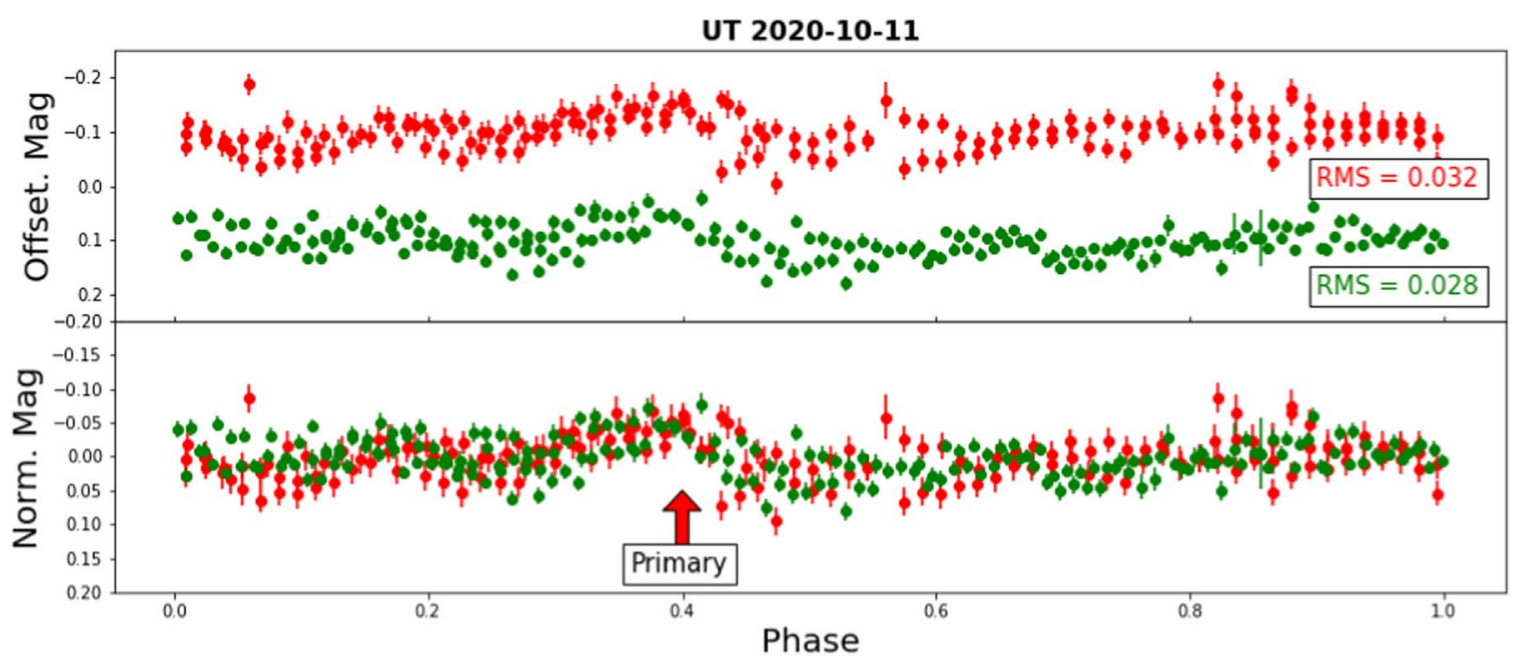

Figure 17. Phase-folded lightcurve of Gault on 2020 October 11 UT from MLO and LOT folded by $2.5 \mathrm{hr}$. Both lightcurves show a strong primary peak but neither appear to show a strong secondary peak.

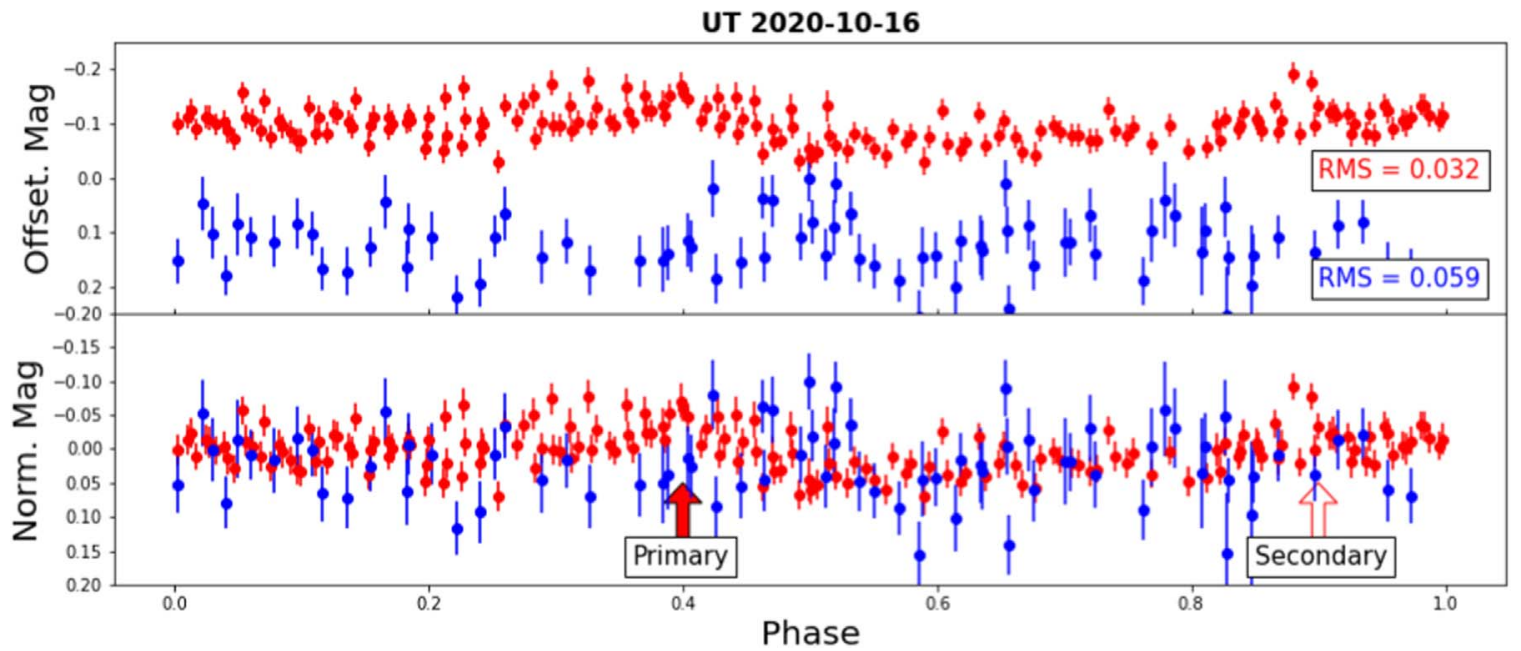

Figure 18. Phase-folded lightcurve of Gault on 2020 October 16 UT from MLO and GIT folded by $2.5 \mathrm{hr}$. Unfortunately, the GIT observations were affected by lessthan-ideal seeing on this date (3!" 1 ; see Table 2), and therefore, it is difficult to see either peak through the noise. The MLO data show possible primary and secondary peaks but are also affected by noise.

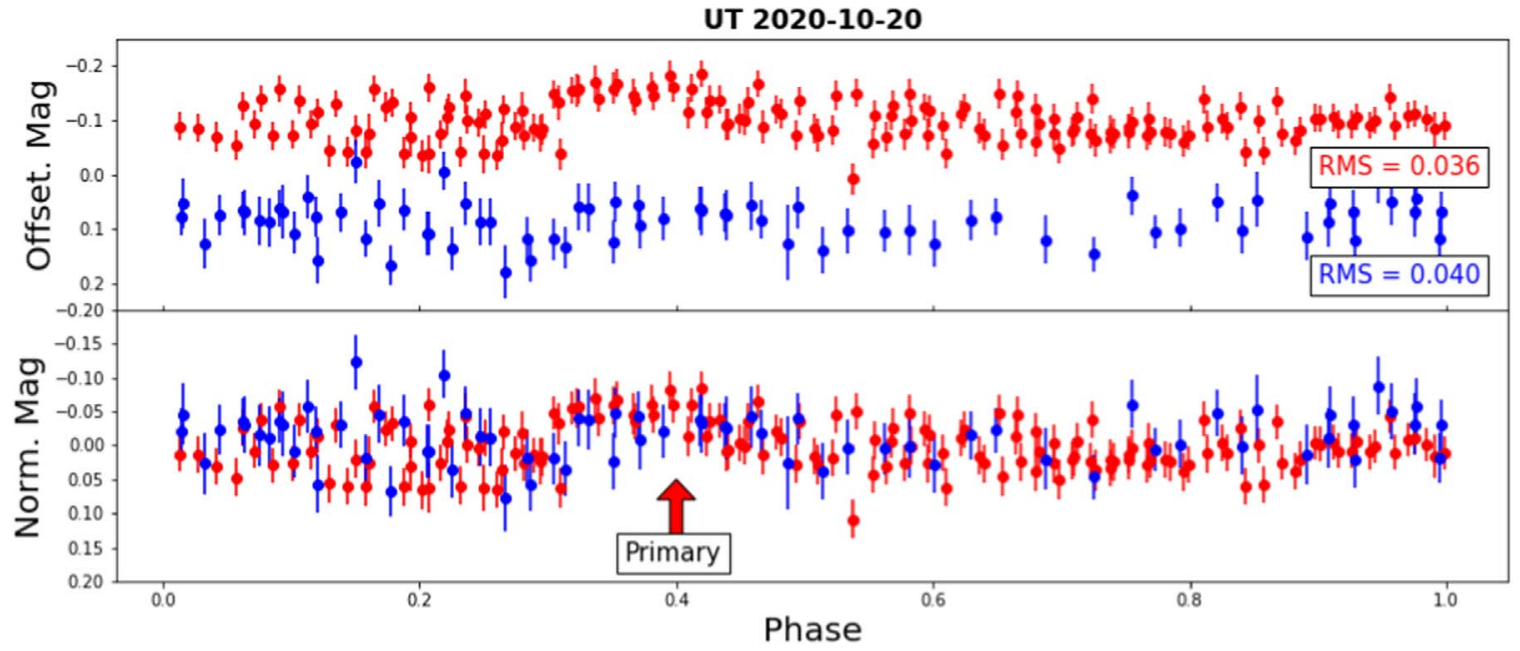

Figure 19. Phase-folded lightcurve of Gault on 2020 October 20 UT from MLO and GIT folded by 2.5 hr. Both lightcurves show promising primary peaks but do not display strong secondary peaks. 


\section{ORCID iDs}

Josiah N. Purdum (10 https://orcid.org/0000-0003-1227-3738 Zhong-Yi Lin (1) https://orcid.org/0000-0003-3827-8991 Bryce T. Bolin (i) https://orcid.org/0000-0002-4950-6323 Kritti Sharma iㅣ https://orcid.org/0000-0002-4477-3625 Varun Bhalerao (ib https://orcid.org/0000-0002-6112-7609 Josef Hanuš (1) https://orcid.org/0000-0002-2934-3723 Harsh Kumar iㅏ https://orcid.org/0000-0003-0871-4641 Robert Quimby (1) https://orcid.org/0000-0001-9171-5236 Joannes C. van Roestel (1) https://orcid.org/0000-00022626-2872

Chengxing Zhai 10 https://orcid.org/0000-0002-0291-4522 Yanga R. Fernandez (i) https://orcid.org/0000-00031156-9721

Carey M. Lisse (10) https://orcid.org/0000-0002-9548-1526 Dennis Bodewits (1) https://orcid.org/0000-0002-2668-7248 Christoffer Fremling (1) https://orcid.org/0000-00024223-103X

Nathan Ryan Golovich (1) https://orcid.org/0000-00032632-572X

Chen-Yen Hsu (i) https://orcid.org/0000-0002-8390-3082

Chow-Choong Ngeow (1) https://orcid.org/0000-00018771-7554

Yuhan Yao iㅏ https://orcid.org/0000-0001-6747-8509 Tomás Ahumada (1) https://orcid.org/0000-0002-2184-6430 Shreya Anand (1) https://orcid.org/0000-0003-3768-7515 Igor Andreoni (1) https://orcid.org/0000-0002-8977-1498 Kevin B. Burdge (10) https://orcid.org/0000-0002-7226-836X Chan-Kao Chang (i) https://orcid.org/0000-0003-1656-4540 Chris M. Copperwheat (1) https://orcid.org/0000-00017983-8698

Michael Coughlin (10 https://orcid.org/0000-0002-8262-2924 Richard Dekany (1) https://orcid.org/0000-0002-5884-7867 Dmitry Duev (i) https://orcid.org/0000-0001-5060-8733 Matthew Graham (i) https://orcid.org/0000-0002-3168-0139 Erik C. Kool (1) https://orcid.org/0000-0002-7252-3877 Mansi M. Kasliwal (i) https://orcid.org/0000-0002-5619-4938 Shrinivas R. Kulkarni (ib https://orcid.org/0000-00015390-8563

Russ R. Laher (1) https://orcid.org/0000-0003-2451-5482 Ashish Mahabal (1) https://orcid.org/0000-0003-2242-0244 Frank J. Masci ๑ https://orcid.org/0000-0002-8532-9395 Przemyslaw J. Mróz iㅏ https://orcid.org/0000-00017016-1692

Reed Riddle (1) https://orcid.org/0000-0002-0387-370X

Roger M. Smith (1) https://orcid.org/0000-0001-7062-9726

Lin Yan (ㄷ) https://orcid.org/0000-0003-1710-9339

\section{References}

Astropy Collaboration, Price-Whelan, A. M., \& Sipőcz, B. M. 2018, AJ, 156,123

Astropy Collaboration, Robitaille, T. P., \& Tollerud, E. J. 2013, A\&A, 558, A33

Bellm, E. C., Kulkarni, S. R., Graham, M. J., et al. 2019, PASP, 131, 018002 Bertin, E. 2011, in ASP Conf. Ser. 442, Astronomical Data Analysis Software and Systems XX, ed. I. N. Evans et al. (San Francisco, CA: ASP), 435

Binzel, R. P., Farinella, P., Zappalà, V., \& Cellino, A. 1989, in Asteroids II, ed. R. P. Binzel, T. Gehrels, \& M. S. Matthews (Tucson, AZ: Univ. Arizona Press), 416

Bolin, B., Jedicke, R., Granvik, M., et al. 2014, Icar, 241, 280

Bolin, B. T., Fernandez, Y. R., Lisse, C. M., et al. 2021, AJ, 161, 116
Bolin, B. T., Fremling, C., Holt, T. R., et al. 2020a, ApJL, 900, L45 Bolin, B. T., \& Lisse, C. M. 2020, MNRAS, 497, 4031

Bolin, B. T., Lisse, C. M., Kasliwal, M. M., et al. 2020b, AJ, 160, 26 Bolin, B. T., Weaver, H. A., Fernandez, Y. R., et al. 2018, ApJL, 852, L2

Bottke, W. F., Jr., Vokrouhlickỳ, D., Rubincam, D. P., \& Nesvornỳ, D. 2006, AREPS, 34, 157

Bowell, E., Hapke, B., Domingue, D., et al. 1989, in Asteroids II, ed. R. P. Binzel, T. Gehrels, \& M. S. Matthews (Tucson, AZ: Univ. Arizona Press), 524

Carbognani, A., \& Buzzoni, A. 2020, MNRAS, 493, 70

Carry, B. 2012, P\&SS, 73, 98

Chambers, K., Magnier, E., Metcalfe, N., et al. 2016, arXiv:1612.05560

Chandler, C. O., Kueny, J., Gustafsson, A., et al. 2019, ApJL, 877, L12

Chang, C.-K., Lin, H.-W., Ip, W.-H., et al. 2016, ApJS, 227, 20

Chang, C.-K., Lin, H.-W., Ip, W.-H., et al. 2017, ApJL, 840, L22

Chang, C.-K., Lin, H.-W., Ip, W.-H., et al. 2019, ApJS, 241, 6

Dekany, R., Smith, R. M., Riddle, R., et al. 2020, PASP, 132, 038001

Delbo, M., Libourel, G., Wilkerson, J., et al. 2014, Natur, 508, 233

Farinella, P., Vokrouhlický, D., \& Hartmann, W. K. 1998, Icar, 132, 378

Ferrín, I., Fornari, C., \& Acosta, A. 2019, MNRAS, 490, 219

Flewelling, H. 2018, AAS Meeting Abstracts, 231, 436.01

Graham, M. J., Kulkarni, S. R., Bellm, E. C., et al. 2019, PASP, 131, 078001

Hainaut, O., Boehnhardt, H., Snodgrass, C., et al. 2014, A\&A, 563, A75

Hanuš, J., Delbo, M., Alí-Lagoa, V., et al. 2018, Icar, 299, 84

Harding, L. K., Hallinan, G., Milburn, J., et al. 2016, MNRAS, 457, 3036

Harris, A., Pravec, P., Galád, A., et al. 2014, Icar, 235, 55

Hirabayashi, M., Nakano, R., Tatsumi, E., et al. 2020, Icar, 352, 113946

Huehnerhoff, J., Ketzeback, W., Bradley, A., et al. 2016, Proc. SPIE, 9908, $99085 \mathrm{H}$

Hui, M.-T., Kim, Y., \& Gao, X. 2019, MNRAS: Letters, 488, L143

Ivanova, O., Skorov, Y., Luk'yanyk, I., et al. 2020, MNRAS, 496, 2636

Jedicke, R., Bolin, B., Granvik, M., \& Beshore, E. 2016, Icar, 266, 173

Jewitt, D., Agarwal, J., Li, J., et al. 2017a, AJ, 153, 223

Jewitt, D., Agarwal, J., Weaver, H., Mutchler, M., \& Larson, S. 2013, ApJL, 778, L21

Jewitt, D., Asmus, D., Yang, B., \& Li, J. 2019a, AJ, 157, 193

Jewitt, D., Hsieh, H., \& Agarwal, J. 2015, in Asteroids IV, ed. P. Michel, F. E. DeMeo, \& W. F. BOttke (Tucson, AZ: Univ. Arizona Press)

Jewitt, D., Kim, Y., Luu, J., et al. 2019b, ApJL, 876, L19

Jewitt, D., Luu, J., Rajagopal, J., et al. 2017b, ApJL, 850, L36

Kasliwal, M. M., Cannella, C., Bagdasaryan, A., et al. 2019, PASP, 131, 038003

Kelley, M. S. P., Bodewits, D., Ye, Q., et al. 2019, ZChecker: Zwicky Transient Facility Moving Target Checker for Short Object Lists, version 2.6.1, Astrophysics Source Code Library, ascl:1907.017

Kim, M.-J., Lee, H.-J., Lee, S.-M., et al. 2018, A\&A, 619, A123

Kleyna, J. T., Hainaut, O. R., Meech, K. J., et al. 2019, ApJL, 874, L20

Laher, R. R., Gorjian, V., Rebull, L. M., et al. 2012, PASP, 124, 737

Lang, D., Hogg, D. W., Mierle, K., Blanton, M., \& Roweis, S. 2010, AJ, 139,1782

Lomb, N. R. 1976, Ap\&SS, 39, 447

Marsset, M., DeMeo, F., Sonka, A., et al. 2019, ApJL, 882, L2

Masci, F. J., Laher, R. R., Rusholme, B., et al. 2019, PASP, 131, 018003

McCully, C., \& Tewes, M. 2019, Astro-SCRAPPY: Speedy Cosmic Ray Annihilation Package in Python, version 1.0.5, Astrophysics Source Code Library, ascl:1907.032

Moreno, F., Jehin, E., Licandro, J., et al. 2019, A\&A, 624, L14

Moreno, F., Pozuelos, F. J., Novaković, B., et al. 2017, ApJL, 837, L3

Muinonen, K., Belskaya, I. N., Cellino, A., et al. 2010, Icar, 209, 542

Pravec, P., Harris, A. W., Kušnirák, P., Galád, A., \& Hornoch, K. 2012, Icar, 221,365

Pravec, P., Harris, A. W., Vokrouhlický, D., et al. 2008, Icar, 197, 497

Press, W. H., Flannery, B. P., \& Teukolsky, S. A. 1986, Numerical Recipes. The Art of Scientific Computing (Cambridge: Cambridge Univ. Press)

Purdum, J., Lin, Z.-Y., Bolin, B. T., et al. 2020, ATel, 13885, 1

Sanchez, J. A., Reddy, V., Thirouin, A., et al. 2019, ApJL, 881, L6

Sánchez, P., \& Scheeres, D. J. 2014, M\&PS, 49, 788

Scheeres, D. 2015, Icar, 247, 1

Smith, K., Denneau, L., Vincent, J.-B., \& Weryk, R. 2019, CBET, 4594, 1 Snodgrass, C., Tubiana, C., Vincent, J.-B., et al. 2010, Natur, 467, 814

Taylor, P. A., Rivera-Valentín, E. G., Benner, L. A., et al. 2019, P\&SS, 167, 1 Vernazza, P., \& Beck, P. 2016, arXiv:1611.08731

Vokrouhlický, D., Pravec, P., Durech, J., et al. 2017, A\&A, 598, A91

Ye, Q., Kelley, M. S. P., Bodewits, D., et al. 2019, ApJL, 874, L16 